\title{
Comments on lump solutions in SFT
}

\author{
Loriano Bonora $^{1,2, a}$, Driba D. Tolla ${ }^{1,2, b}$ \\ ${ }^{1}$ International School for Advanced Studies (SISSA), Via Bonomea 265, 34136 Trieste, Italy \\ 2 INFN, Sezione di Trieste, Trieste, Italy
}

Received: 15 December 2014 / Accepted: 5 April 2016 / Published online: 13 April 2016

(C) The Author(s) 2016. This article is published with open access at Springerlink.com

\begin{abstract}
We analyze a recently proposed scheme to construct analytic lump solutions in open SFT. We argue that in order for the scheme to be operative and to guarantee background independence it must be implemented in the same 2D conformal field theory in which SFT is formulated. We outline and discuss two different possible approaches. Next we reconsider an older proposal for analytic lump solutions and implement a few improvements. In the course of the analysis we formulate a distinction between regular and singular gauge transformations and advocate the necessity of defining a topology in the space of string fields.
\end{abstract}

\section{Introduction}

After the discovery $[1,2]$ of the first analytic solution of open SFT à la Witten [3], which links the perturbative vacuum to the tachyon vacuum, there have been a considerable number of papers devoted to related solutions [4,5] and to marginal deformations thereof [6-13]. The literature concerning analytic lump solutions, i.e. analytic solutions interpretable as lower dimensional branes (meant to complete the analytic proof of the three conjectures by Sen [14]), is instead poorer. There have been essentially two attempts to find such analytic solutions: the first is the so-called BMT proposal [1517], the second is the most recent one formulated in [18], which we will refer to as EM. They are both modeled on the Erler-Schnabl (ES) solution [5], an alternative simpler formulation of the original tachyon vacuum (TV) solution (for recent reviews on the whole subject, see [19-22]). In [18] the construction is based on previous results on correlators involving boundary condition changing (bcc) operators and on a set of (implicit) prescriptions laid down in order for the solution to satisfy the SFT equation of motion. As we shall see, hidden behind this is the risk of background depen- dence. More precisely, we wish to clarify first of all if and to what extent these results and prescriptions can be embedded in SFT; second, we would like to discuss their background independence. By the latter we mean that one can derive a variety of backgrounds as solutions of the SFT equation of motion, although such an equation of motion is formulated in a specific background (the D25-brane). The only way to clarify these issues is to implement the EM approach in an explicit 2D field theory formulation similar to the one in $[5,12,15]$, consistent with the original one in which the SFT is formulated. The first part of our paper is an attempt in this direction. We clarify the problems behind the EM proposal and try to solve them. We do not succeed in carrying out this task, but, nevertheless, we think our scrutiny may be instructive. More explicitly, while we believe the first issue above, although not solved by us in this paper, should anyhow be implementable, the second, i.e. background independence, looks almost impossible to realize.

We then turn to the BMT proposal and, in the second part of the paper, we discuss some aspects of the latter and present a few improvements. In the course of the analysis we propose a definite distinction between singular and regular BRST transformations, which turns out to be instrumental in clarifying some confused issues present in analytic solutions of SFT.

The paper is organized as follows. Sections 2, 3, and 6 are short introductions to the ES solution and to the EM and BMT proposals. Section 4 is devoted to a detailed presentation of the bcc operators used in the EM proposal. Section 5 contains our (failed) attempt to implement the EM approach in an explicit field (oscillator) formulation. Section 7 contains a digression on gauge transformations and identity based solutions, which is needed for the subsequent developments in Sect. 8, where they are applied to the BMT proposal. Section 9 contains some conclusions.

\footnotetext{
a e-mail: bonora@sissa.it

be-mail:dribatolla@gmail.com
} 


\section{Short review of the Erler-Schnabl solution}

Even though the content of this section is very well known, we nevertheless briefly review the classical solution and its properties found by Erler and Schnabl [5], given its paradigmatic role with respect to the proposed lump solutions. To start with let us introduce the $\{K, B, c\}$ algebra, where

$K=\frac{\pi}{2} K_{1}^{L}|I\rangle, \quad B=\frac{\pi}{2} B_{1}^{L}|I\rangle, \quad c=c(1)|I\rangle$,

where the argument of the $c$ ghost field refers to the arctan frame and

$$
\begin{aligned}
K_{1}^{L}= & \frac{1}{2} K_{1}+\frac{1}{\pi}\left(\mathcal{L}_{0}+\mathcal{L}_{0}^{\dagger}\right), \\
& B_{1}^{L}=\frac{1}{2} B_{1}+\frac{1}{\pi}\left(\mathcal{B}_{0}+\mathcal{B}_{0}^{\dagger}\right),
\end{aligned}
$$

and the $K_{1}, \mathcal{L}_{0}$, and $B_{1}, \mathcal{B}_{0}$ are expressed in terms of the world-sheet energy-momentum tensor and the $b$ ghost field in the following way:

$$
\begin{aligned}
& \mathcal{L}_{0}= L_{0}+\sum_{k=1}^{\infty} \frac{2(-1)^{k+1}}{4 k^{2}-1} L_{2 k}, \\
& \mathcal{B}_{0}=b_{0}+\sum_{k=1}^{\infty} \frac{2(-1)^{k+1}}{4 k^{2}-1} b_{2 k}, \\
& K_{1}=L_{1}+L_{-1}, \quad B_{1}=b_{1}+b_{-1} .
\end{aligned}
$$

Then the string fields $K, B$, and $c$ generate an algebra with the following commutation relations and BRST variations:

$$
\begin{aligned}
& {[K, B]=0, \quad\{B, c\}=1, \quad[K, c]=\partial c, \quad\{B, \partial c\}=0,} \\
& B^{2}=0=c^{2}, \quad Q B=K, \quad Q K=0, \\
& Q c=c K c=c \partial c .
\end{aligned}
$$

In the above the juxtaposition of any two symbols represents the star product, whose symbol has been understood. The commutators are taken with respect to the star product.

The Erler-Schnabl (ES) solution is given by

$\psi_{E S}=c B(K+1) c \frac{1}{K+1}$.

The solution can be formally written via a singular gauge transformation of the perturbative vacuum (see $[4,23]$ )

$\psi_{E S}=U_{0} Q U_{0}^{-1}$,

with

$U_{0}=1-\frac{1}{K+1} B c$ and $U_{0}^{-1}=1+\frac{1}{K} B c$.

In order to show that the ES solution satisfies Sen's first conjecture one computes the energy corresponding to it. The energy density is (in the sequel we set $g_{o}=1$ )

$$
\begin{aligned}
E\left[\psi_{E S}\right]= & \frac{1}{6}\left\langle\psi_{0}, Q \psi_{0}\right\rangle \\
= & \frac{1}{6}\left\langle(c+c K B c) \frac{1}{K+1} c K c \frac{1}{K+1}\right\rangle \\
= & \frac{1}{6}\left\langle c \frac{1}{K+1} c K c \frac{1}{K+1}\right\rangle \\
& -\left\langle Q\left(B c \frac{1}{K+1} c K c \frac{1}{K+1}\right)\right\rangle .
\end{aligned}
$$

The last term vanishes since it is BRST exact and the first term in the second line gives

$$
\begin{aligned}
E\left[\psi_{E S}\right] & =\frac{1}{6} \int_{0}^{\infty} \mathrm{d} t_{1} \mathrm{~d} t_{2} e^{-t_{1}-t_{2}}\left\langle c e^{-t_{1} K} c \partial c e^{-t_{2} K}\right\rangle_{C_{t_{1}+t_{2}}} \\
& =-\frac{1}{6} \int_{0}^{\infty} \mathrm{d} t_{1} \mathrm{~d} t_{2} e^{-t_{1}-t_{2}} \frac{\left(t_{1}+t_{2}\right)^{2}}{\pi^{2}} \sin ^{2}\left(\frac{\pi t_{1}}{t_{1}+t_{2}}\right) \\
& =-\frac{1}{2 \pi^{2}}
\end{aligned}
$$

where $C_{t}$ denotes a cylinder in the arctan frame of circumference $t$, and we have used the Schwinger parametrization

$\frac{1}{1+K}=\int_{0}^{\infty} e^{-t(1+K)}$.

In passing from the first line to the second line of (8) one starts from the correlator $\left\langle c\left(z_{1}\right) c \partial c\left(z_{2}\right)\right\rangle=-\left(z_{1}-z_{2}\right)^{2}$ in the upper half-plane, and maps it to the arctan frame via the $\operatorname{map} \xi=\arctan (z)$, so that it becomes

$\left\langle c\left(\xi_{1}\right) c \partial c\left(\xi_{2}\right)\right\rangle=-\sin ^{2}\left(\xi_{1}-\xi_{2}\right)$

in the cylinder $C_{\pi}$. Finally one rescales $\xi \rightarrow \frac{\ell}{\pi} \xi$ in order to map to a cylinder $C_{\ell}$.

Equation (8) means that the ES solution correctly reproduces minus the $D 25$-brane tension, thus identifies the tachyon condensation vacuum. The Erler-Schnabl solution does not support open string states, i.e. this solution agrees with Sen's second conjecture. A simple way to show that a given solution $\psi_{0}$ does not support open string states is to find a homotopy operator $\mathcal{A}$ such that

$\mathcal{Q}_{\psi_{0}} \mathcal{A}=1$,

with

$\mathcal{Q}_{\psi_{0}} \mathcal{A}=Q \mathcal{A}+\psi_{0} \mathcal{A}+\mathcal{A} \psi_{0}$

for, if $\mathcal{Q}_{\psi_{0}} \phi=0$ this implies that $\phi=\mathcal{Q}_{\psi_{0}} \chi$ with $\chi=\mathcal{A} \phi$. For the ES solution, the homotopy operator exists and has the form [2]

$\mathcal{A}=B \frac{1}{1+K}$. 


\section{The EM proposal}

Let us briefly review here the recent EM proposal, at least for the (essential) aspects we wish to discuss. The EM proposal is constructed by introducing in the ES solution boundary condition changing (bcc) operators, in analogy to the solution of Kiermaier, Okawa, and Soler (KOS), for marginal deformations (see [12] and below). The KOS solution is written in terms of boundary condition changing (bcc) operators $\sigma$ and $\bar{\sigma}$ satisfying the following OPE:

$\sigma \bar{\sigma}=\bar{\sigma} \sigma=1$

In EM the relevant bcc operators are not the same as in KOS, of course, rather they change the string boundary conditions from $N$ to $D$ and vice versa. The property (12) is crucial for the solution to satisfy the equation of motion and, at first, it seems difficult to extend the procedure to solutions which describe BCFTs that are not connected to the original BCFT by a marginal deformation. The reason is that generic bcc operators relating two BCFTs have non-trivial OPE and cannot satisfy (12). In order to overcome this difficulty the authors of [18] tensor those bcc operators with plane-wave factors and introduce modified bcc operators as follows:

$\sigma(s)=\sigma_{*}(s) e^{i \sqrt{h} X^{0}(s)}, \quad \bar{\sigma}(s)=\bar{\sigma}_{*}(s) e^{-i \sqrt{h} X^{0}(s)}$,

where $\sigma_{*}(s)$ and $\bar{\sigma}_{*}(s)$ are the bcc operators relating the two BCFTs and they are primary operators of conformal dimension $h$. In particular, for the lump solution they are given by $\sigma_{*}=\sigma_{N D}, \bar{\sigma}_{*}=\sigma_{D N}$ and $h=\frac{1}{16}$. The modified bcc operators satisfy

$\sigma \bar{\sigma}=$ finite,$\quad \bar{\sigma} \sigma=1$

EM assumes that the BRST variations of the modified bcc operators are

$Q \sigma=c \partial \sigma=c[K, \sigma], \quad Q \bar{\sigma}=c \partial \bar{\sigma}=c[K, \bar{\sigma}]$

where in [18] $Q$ is the BRST operator of the original D25brane BCFT. This is one of the critical aspects we must discuss. In fact, as we shall see, there is no a priori guarantee that the action of $Q$ is well defined on $\sigma, \bar{\sigma}$.

Continuing the presentation of the EM proposal, the EM solution for the equation of motion at the tachyon vacuum is given by

$\Phi_{0}=-\Sigma \Psi_{0} \bar{\Sigma}$ where

$$
\begin{aligned}
\Psi_{0} & =\frac{1}{\sqrt{1+K}} c(1+K) B c \frac{1}{\sqrt{1+K}}, \\
\Sigma & =\mathcal{Q}\left(\frac{1}{\sqrt{1+K}} B \sigma \frac{1}{\sqrt{1+K}}\right), \\
\bar{\Sigma} & =\mathcal{Q}\left(\frac{1}{\sqrt{1+K}} B \bar{\sigma} \frac{1}{\sqrt{1+K}}\right) .
\end{aligned}
$$

Here $\mathcal{Q}=Q+\left[\Psi_{0},\right]$ is the BRST operator at the tachyon vacuum. Explicitly, the proposed solution is given by

$$
\Phi_{0}=-\frac{1}{\sqrt{1+K}} c(1+K) \sigma \frac{B}{1+K} \bar{\sigma}(1+K) c \frac{1}{\sqrt{1+K}} .
$$

The solution to the equation of motion at the perturbative vacuum is

$\Psi=\Psi_{0}+\Phi_{0}$

and its energy is given by

$$
\begin{aligned}
E & =-S[\Psi]=-\frac{g_{0}}{2 \pi^{2}}+\frac{1}{6} \operatorname{Tr}\left[\Phi_{0}^{3}\right]=-\frac{g_{0}}{2 \pi^{2}}-\frac{1}{6} \operatorname{Tr}\left[\left(\Psi_{0}\right)^{3}\right] \\
& =-\frac{g_{0}}{2 \pi^{2}}+\frac{g_{*}}{2 \pi^{2}} .
\end{aligned}
$$

Using previous results in the literature, in particular [29], the authors of [18] were able to show that $g_{*}-g_{0}$ is consistent with the difference between the tension of a D25- and a D24brane.

In the EM ansatz many details are understood, and we must ask: Is it possible to implement this formulation in a concrete field theory formalism, consistent with that of SFT? ${ }^{1}$

In the following we would like to bring to light the hidden details and assess their validity. To start with, two BCFT's are mentioned in [18], $\mathrm{BCFT}_{0}$ and $\mathrm{BCFT}_{*}$, and a rule is declared according to which, when writing the solution, any operator that appears to the left of $\sigma$ or to the right of $\bar{\sigma}$ belongs to $\mathrm{BCFT}_{0}$, while operators which appear to the right of $\sigma$ or to the left of $\bar{\sigma}$ belong to $\mathrm{BCFT}_{*}$, but no distinction is made to specify what string fields belong to the former and what to the latter. It may well be that the $Q$ and $K$ that are defined on $\mathrm{BCFT}_{0}$ are the same that live on $\mathrm{BCFT}_{*}$ (this is one of the hypotheses we will consider later on). But this is a crucial

\footnotetext{
1 This is what we deem necessary. Not everybody shares this opinion. The referee of this paper believes that this problem we think to be so important is already considered and implicitly solved in the first ref. [38]; see eqs. (8.13-14) there. We take note of this, but we believe that just because of this it should be possible to formulate the EM proposal in a more explicit form using the oscillator formalism. In the process the issue of background dependence or independence will automatically emerge.
} 
aspect that must be carefully justified. As we will see more clearly later on, the $\mathrm{BCFT}_{0}$ is the $\mathrm{BCFT}$ with $\mathrm{NN}$ boundary conditions for all directions, while $\mathrm{BCFT}_{*}$ is characterized by DD boundary conditions along one space direction, the 25th, say, and NN along the remaining ones. To keep track of this we will simply label $Q$ and $K$ in these theories with $\mathrm{NN}$ and DD, respectively.

Thus, in order to be consistent with the EM rules, when passing through $\sigma$, operators like $Q_{\mathrm{NN}}$ and $K_{\mathrm{NN}}$, become $Q_{\mathrm{DD}}$ and $K_{\mathrm{DD}}$, and they switch back to $Q_{\mathrm{NN}}$ and $K_{\mathrm{NN}}$ when they pass through $\bar{\sigma}$. We will discuss later on how it is possible to make sense of such switching of operators. In the rest of this section we would like to make it evident that considering only two BCFTs, $\mathrm{BCFT}_{0}$, and $\mathrm{BCFT}_{*}$, is not enough.

In a first attempt to write the EM proposal (16) in a more precise way, we rewrite it as

$\Phi_{0}=-\Sigma \Psi_{0}^{*} \bar{\Sigma}$

where

$$
\begin{aligned}
\Psi_{0}^{*} & =\frac{1}{\sqrt{1+K_{\mathrm{DD}}}} c\left(1+K_{\mathrm{DD}}\right) B c \frac{1}{\sqrt{1+K_{\mathrm{DD}}}}, \\
\Sigma & =\mathcal{Q}_{\mathrm{NN}}\left(\frac{1}{\sqrt{1+K_{\mathrm{NN}}}} B \sigma \frac{1}{\sqrt{1+K_{\mathrm{DD}}}}\right), \\
\bar{\Sigma} & =\mathcal{Q}_{\mathrm{DD}}\left(\frac{1}{\sqrt{1+K_{\mathrm{DD}}}} B \bar{\sigma} \frac{1}{\sqrt{1+K_{\mathrm{NN}}}}\right)
\end{aligned}
$$

with $\mathcal{Q}_{\mathrm{NN}}=Q_{\mathrm{NN}}+\left[\Psi_{0},\right]$ and $\mathcal{Q}_{\mathrm{DD}}=Q_{\mathrm{DD}}+\left[\Psi_{0}^{*}\right.$, ] are the BRST operators at the tachyon vacuum. Explicitly, the ansatz is given by

$$
\begin{aligned}
\Phi_{0}= & -\frac{1}{\sqrt{1+K_{\mathrm{NN}}}} c\left(1+K_{\mathrm{NN}}\right) \sigma \frac{B}{1+K_{\mathrm{DD}}} \\
& \times \bar{\sigma}\left(1+K_{\mathrm{NN}}\right) c \frac{1}{\sqrt{1+K_{\mathrm{NN}}}} .
\end{aligned}
$$

The solution to the equation of motion at the perturbative vacuum is

$\Psi=\Psi_{0}+\Phi_{0}$

where $\Psi_{0}=\frac{1}{\sqrt{1+K_{\mathrm{NN}}}} c\left(1+K_{\mathrm{NN}}\right) B c \frac{1}{\sqrt{1+K_{\mathrm{NN}}}}$ is still the ES TV solution, and its energy is expected to yield

$$
\begin{aligned}
E & =-S[\Psi]=-\frac{g_{0}}{2 \pi^{2}}+\frac{1}{6} \operatorname{Tr}\left[\Phi_{0}^{3}\right] \\
& =-\frac{g_{0}}{2 \pi^{2}}-\frac{1}{6} \operatorname{Tr}\left[\left(\Psi_{0}^{*}\right)^{3}\right]=-\frac{g_{0}}{2 \pi^{2}}+\frac{g_{*}}{2 \pi^{2}} .
\end{aligned}
$$

Now that we have heuristically rewritten the EM rules in a more explicit form let us point out some difficulties. The fact that the EM ansatz yields the expected result depends crucially on enlarging the $K, B, c$ algebra by including $\sigma$ and $\bar{\sigma}$. The $K, B, c$ algebras (for zero momentum states) for the NN and DD cases are identical (see below). For clarity let us write them once more

$\{B, c\}=0, \quad\{B, \partial c\}=0, \quad\left[K_{i i}, B\right]=0, \quad\left[K_{i i}, c\right]=\partial c$,

where $i i$ denotes either NN or DD. The action of the BRST operators on those quantities should mimic Eq. (3),

$Q_{i i} B=K_{i i}, \quad Q_{i i} K_{i i}=0, \quad Q_{i i} c=c K_{i i} c$.

From Eq. (1) we directly get the representation of two of the elements of the algebra (26), but the representation of $K_{\mathrm{DD}}$ should be obtained as the BRST variation of $B$ :

$$
\begin{aligned}
\frac{\pi}{2} Q_{\mathrm{DD}} B_{1}^{L}|I\rangle & =\frac{\pi}{2}\left\{Q_{\mathrm{DD}}, B_{1}^{L}\right\}|I\rangle-\frac{\pi}{2} B_{1}^{L} Q_{\mathrm{DD}}|I\rangle \\
& =\frac{\pi}{2} K_{1, \mathrm{DD}}^{L}|I\rangle-\frac{\pi}{2} B_{1}^{L} Q_{\mathrm{DD}}|I\rangle .
\end{aligned}
$$

So we see that the BRST operator $Q_{\mathrm{DD}}$ must annihilate the identity state $|I\rangle$ or any wedge state in general, otherwise the definition of $K_{\mathrm{DD}}$ as a BRST variation of $B$ cannot be realized at the representation level. Is this the case? The answer is not obvious.

Another issue of concern is related to the computation of physical observables for the solution $\Psi_{0}^{*}$. In order to calculate the energy or the closed string overlap, one needs to use the following Schwinger parametrization for the inverse of $1+K_{\mathrm{DD}}$ :

$\frac{1}{1+K_{\mathrm{DD}}}=\int_{0}^{\infty} \mathrm{d} t e^{-t} e^{-t K_{\mathrm{DD}}}$.

In the ES solution, we replace $e^{-t K}$ by $\Omega^{t}$, where $\Omega$ is the $\operatorname{SL}(2, \mathbb{R})$ invariant vacuum, which defines the wedge states. Now, in the presence of the bcc operators, is the vacuum $\operatorname{SL}(2, \mathbb{R})$ invariant and does the new vacuum have the right properties to define new wedge states?

A third problem arises when we try to verify the equation of motion for (23). In our notation the equation of motion at the TV is

$\mathcal{Q}_{\mathrm{NN}} \Phi+\Phi^{2}=0 \Rightarrow Q_{\mathrm{NN}} \Phi+\left\{\Psi_{0}, \Phi\right\}+\Phi^{2}=0$.

According to the EM rules rewritten above, the BRST operator acts on $\Phi_{0}$ as follows:

$$
\begin{aligned}
Q_{\mathrm{NN}} \Phi_{0}= & -\frac{1}{\sqrt{1+K_{\mathrm{NN}}}}\left(Q_{\mathrm{NN}} c\right)\left(1+K_{\mathrm{NN}}\right) \sigma \frac{B}{1+K_{\mathrm{DD}}} \\
& \times \bar{\sigma}\left(1+K_{\mathrm{NN}}\right) c \frac{1}{\sqrt{1+K_{\mathrm{NN}}}} \\
& +\frac{1}{\sqrt{1+K_{\mathrm{NN}}}} c\left(1+K_{\mathrm{NN}}\right)(Q \sigma) \frac{B}{1+K_{\mathrm{DD}}}
\end{aligned}
$$




$$
\begin{aligned}
& \times \bar{\sigma}\left(1+K_{\mathrm{NN}}\right) c \frac{1}{\sqrt{1+K_{\mathrm{NN}}}} \\
& +\frac{1}{\sqrt{1+K_{\mathrm{NN}}}} c\left(1+K_{\mathrm{NN}}\right) \sigma \frac{\left(Q_{\mathrm{DD}} B\right)}{1+K_{\mathrm{DD}}} \\
& \times \bar{\sigma}\left(1+K_{\mathrm{NN}}\right) c \frac{1}{\sqrt{1+K_{\mathrm{NN}}}} \\
& -\frac{1}{\sqrt{1+K_{\mathrm{NN}}}} c\left(1+K_{\mathrm{NN}}\right) \\
& \times \sigma \frac{B}{1+K_{\mathrm{DD}}}(Q \bar{\sigma})\left(1+K_{\mathrm{NN}}\right) c \frac{1}{\sqrt{1+K_{\mathrm{NN}}}} \\
& -\frac{1}{\sqrt{1+K_{\mathrm{NN}}}} c\left(1+K_{\mathrm{NN}}\right) \\
& \times \sigma \frac{B}{1+K_{\mathrm{DD}}} \bar{\sigma}\left(1+K_{\mathrm{NN}}\right)\left(Q_{\mathrm{NN}} c\right) \frac{1}{\sqrt{1+K_{\mathrm{NN}}}}
\end{aligned}
$$

where $Q \sigma, Q \bar{\sigma}$ is only indicated, but not specified. In fact, in these two cases $Q$ cannot be neither $Q_{\mathrm{NN}}$, nor $Q_{\mathrm{DD}}$. In fact we will see in the next section that $\sigma$ and $\bar{\sigma}$ belong to two additional BCFT's: the BCFT with ND boundary condition along one space direction and the BCFT with DN boundary condition along one space direction (and NN along all the others). The corresponding $Q$ and $K$ will be called $Q_{\mathrm{ND}}, K_{\mathrm{ND}}$, and $Q_{\mathrm{DN}}, K_{\mathrm{DN}}$, respectively.

With these new entries it seems to be more appropriate to rewrite (15) as

$$
\begin{gathered}
Q_{\mathrm{ND}} \sigma=c \partial \sigma=c\left(K_{\mathrm{ND}} \sigma-\sigma K_{\mathrm{ND}}\right), \\
Q_{\mathrm{DN}} \bar{\sigma}=c \partial \bar{\sigma}=c\left(K_{\mathrm{DN}} \bar{\sigma}-\bar{\sigma} K_{\mathrm{DN}}\right) .
\end{gathered}
$$

Assuming these BRST variations of $\sigma$ and $\bar{\sigma}$ and that the star product is consistent with these rules, we notice that (31) contains $K_{\mathrm{ND}}$ and $K_{\mathrm{DN}}$, whereas the other two terms in (30) do not contain such operators. Therefore, the cancellation among those terms is not possible and the equation of motion would not be satisfied.

It is clear that the previous minimalistic cosmetic of the EM rules is too simplistic and only complicates things. A deeper interpretation is necessary. But it is also clear that if the above issues (among others, see below) are not clarified within a concrete field theory formalism, the EM ansatz remains abstract (but see the footnote at the beginning of the section). Our aim in the sequel is to interpret the EM rules in a $2 \mathrm{D}$ conformal field theory context, consistent with the formulation of SFT.

\section{The bcc operator}

One crucial ingredient in the EM ansatz are the bcc operators. We devote this section to a rather detailed description of this subject.
The issue of bcc operators was introduced by Cardy, see [24-26], and subsequently studied and applied by many authors, see in particular [27-30]. It is generally believed that the original and twisted theories are characterized by Hilbert spaces that can be related to each other. This is in a sense trivial, because all countable Hilbert spaces are isomorphic as vector spaces. However, a CFT is not simply characterized by a Hilbert space, but also by the central charge, its primary operators, and by the field theory axioms (locality, for one) and its symmetries. Therefore a direct connection between two such theories in the form of an intertwining operator between the two Hilbert spaces (see below) or, even more, an identification of the two, is far from guaranteed and, if it is possible, it is far from trivial to be determined. This is to stress that it is necessary to analyze in depth the concept and application of the bcc operator, a type of analysis the existing literature does not abound with.

In the sequel, for definiteness, we will use an explicit formulation of the bcc operator, following in particular [27].

The ordinary one-dimensional NN string in the complex $z$ plane is $\left(\alpha^{\prime}=\frac{1}{2}\right)$

$X_{\mathrm{NN}}(z, \bar{z})=x-\frac{i}{2} \alpha_{0}(\ln z+\ln \bar{z})+\frac{i}{2} \sum_{n \neq 0} \frac{\alpha_{n}}{n}\left(z^{-n}+\bar{z}^{-n}\right)$

with $\alpha_{0}=p$, and $\left[\alpha_{m}, \alpha_{n}\right]=m \delta_{n+m}$. The relevant holomorphic propagator is

$\left\langle i \partial X_{\mathrm{NN}}(z) i \partial X_{\mathrm{NN}}(w)\right\rangle \sim \frac{1}{(z-w)^{2}}$.

Then one defines the usual $L_{n}=\frac{1}{2} \sum_{k}: \alpha_{n-k} \alpha_{k}$. The corresponding Virasoro algebra has central charge 1 . The vacuum is defined by $\alpha_{n}|0\rangle=0$ for $n \geq 0$.

On the other hand the one-dimensional DD string is specified by

$$
\begin{aligned}
X_{\mathrm{DD}}(z, \bar{z})= & x_{0}+\frac{i}{2 \pi} \Delta x(\ln z-\ln \bar{z}) \\
& -\frac{i}{2} \sum_{n \neq 0} \frac{\alpha_{n}}{n}\left(z^{-n}-\bar{z}^{-n}\right)
\end{aligned}
$$

where $\Delta x=\left(x_{\pi}-x_{0}\right)$ is the separation between the two Dbranes to which the string is attached. For a single D-brane $\Delta x=0$. The other $\alpha_{n}, n \neq 0$ satisfy the same algebra as the NN string. The holomorphic $U(1)$ current is

$$
i \partial X(z, \bar{z})=-\sum_{n} \alpha_{n} z^{-n-1}
$$

The relevant propagator and the corresponding Virasoro generators have the same form as the NN string. The only difference between the NN and DD cases is the presence of the 
zero mode in the former. ${ }^{2}$ Therefore, the Virasoro algebra is the same for the two cases. The BRST operator is also the same for zero momentum states.

Now we consider the Neumann-Dirichlet (ND) string:

$X_{\mathrm{ND}}(z, \bar{z})=x_{0}+\frac{i}{2} \sum_{r \in \mathbb{Z}+\frac{1}{2}} \frac{\alpha_{r}}{r}\left(z^{-r}+\bar{z}^{-r}\right)$

and the DN one:

$X_{\mathrm{DN}}(z, \bar{z})=x_{0}+\frac{i}{2} \sum_{r \in \mathbb{Z}+\frac{1}{2}} \frac{\alpha_{r}}{r}\left(z^{-r}-\bar{z}^{-r}\right)$.

For later use let us define, according to [27], the (holomorphic) $\mathrm{U}(1)$ current

$J(z)=i \partial X(z)=\sum_{r \in \mathbb{Z}+\frac{1}{2}} \alpha_{r} z^{-r-1}$.

From now on, to avoid misunderstanding, we will replace $\alpha_{r}$ with the symbol $J_{r}$.

Assume the canonical commutator

$\left[J_{r}, J_{s}\right]=r \delta_{r+s}$.

Define the vacuum $\left|\sigma_{*}\right\rangle$ by

$J_{r}\left|\sigma_{*}\right\rangle=0$, for $r>0$

with the corresponding definition of normal ordering. The propagator is

$\langle J(z) J(w)\rangle \sim \frac{1}{(z-w)^{2}}+$ Reg.

The Virasoro generators turn out to be

$\mathcal{L}_{n}=\frac{1}{2} \sum_{r \in \mathbb{Z}+\frac{1}{2}}: J_{r} J_{n-r}:$,
$\mathcal{L}_{0}=a_{0}+\frac{1}{2} \sum_{r \in \mathbb{Z}+\frac{1}{2}} J_{r} J_{-r}$.

Then we find

$\left[\mathcal{L}_{m}, \mathcal{L}_{n}\right]=(m-n) \mathcal{L}_{n+m}+\frac{1}{12}\left(n^{3}-n\right)$

provided $a_{0}=\frac{1}{16}$. It follows that

$\mathcal{L}_{0}\left|\sigma_{*}\right\rangle=\frac{1}{16}\left|\sigma_{*}\right\rangle$.

As usual we conclude that in the $c=1$ CFT there is a primary field $\sigma_{*}(z)$ of weight $\frac{1}{16}$. This is the bcc operator. The first excited state above $\sigma_{*}(z)$ is

$|\tau\rangle=J_{-\frac{1}{2}}\left|\sigma_{*}\right\rangle$.

${ }^{2}$ For the oscillators we use the same symbols in the NN and DD case and in the ND and DN case. The reader is invited to keep in mind the difference.
In terms of the current $J$ we have, for small $z$,

$$
\begin{aligned}
& J(z)\left|\sigma_{*}\right\rangle=z^{-\frac{1}{2}}|\tau\rangle+\mathcal{O}\left(z^{\frac{1}{2}}\right) \\
& J(z)|\tau\rangle=z^{-\frac{3}{2}}\left|\sigma_{*}\right\rangle+z^{-\frac{1}{2}} J_{-\frac{1}{2}}^{2}\left|\sigma_{*}\right\rangle+\mathcal{O}\left(z^{\frac{1}{2}}\right)
\end{aligned}
$$

It is easy to see that $J_{-\frac{1}{2}}^{2}\left|\sigma_{*}\right\rangle=2 \mathcal{L}_{-1}\left|\sigma_{*}\right\rangle=2\left|\sigma_{*}^{\prime}\right\rangle$, and the weight of $\tau$ is $\frac{9}{16}$. There is an infinite towers of such states. The spectrum of these states is completely different from the spectrum of the NN string.

It is clear that we can repeat word by word the same things for the DN string, whose oscillators are also half-integralmode. The vacuum in this case will be denoted by $\left|\bar{\sigma}_{*}\right\rangle$.

In conclusion in the $c=1 \mathrm{CFT}$ there is room for four different Hilbert spaces $\mathcal{H}_{\mathrm{NN}}, \mathcal{H}_{\mathrm{DD}}, \mathcal{H}_{\mathrm{ND}}$, and $\mathcal{H}_{\mathrm{DN}}$. The last two are the same. The first two are also identifiable except for the zero mode $\alpha_{0}$ in the NN case. If the coordinate $X$ is compactified on a circle, the momentum is discrete; dually, in the DD case, we have wrapping modes. In other words the relevant Hilbert spaces are organized in discrete sectors. For the moment let us ignore, for simplicity, such discrete sectors and the momentum in $\mathcal{H}_{\mathrm{NN}}$. We see that, as far as the EM proposal is concerned, we have two kinds of Hilbert spaces, one built out of the integral-mode matter oscillators $\alpha_{n}$ in the 25 th direction, the other with half-integral-mode oscillators $J_{r}$, beside all the other matter and ghost oscillators.

At this point, however, it is worth recalling that OSFT is formulated in terms of NN strings, that is, on the background of the D25-brane. The background independence of OSFT therefore does not rely on the original formulation, but on the fact that, starting from it, we can derive all the other possible backgrounds as analytic solutions of its equation of motion. This is the OSFT's bet. It goes without saying that any background independent solution must be formulated in the original SFT background (the NN string).

As we said above, based on the operator-state correspondence, we can assume that in the $c=1 \mathrm{CFT}$ a primary $\sigma_{*}$ exists of weight $\frac{1}{16}$ (but nonlocal in the conventional sense, [24-26]), such that $\sigma_{*}(0)|0\rangle=\left|\sigma_{*}\right\rangle$, where $|0\rangle$ is the vacuum for the (integral) $\alpha_{n}$ oscillators. However, this is not yet enough to justify the EM rules. There is no guarantee that the field $\sigma_{*}$ admits a free field representation in terms of the free field $X$ or, what is the same, in terms of the free integral oscillators $\alpha_{n}$. If it is true, it must be proven. But if this is not the case we have to give a sense to the operations of applying the SFT operator $Q$ or string field $K$ (which we recall are expressed in terms of free (integral-mode) matter and ghost oscillators) to $\sigma_{*}, \bar{\sigma}_{*}$.

To summarize we therefore face two alternatives. In the best option we can express the fields $\sigma_{*}, \bar{\sigma}_{*}$ in terms of the $\alpha_{n}$ oscillators. In the worst, if this is not possible, we must find a way to deal with different Hilbert spaces. The viability 
of such options is far from obvious and in any case it has not yet been proved.

\section{Two alternatives}

The aim of this section is to find the correct $K, B, c, \sigma_{*}, \bar{\sigma}_{*}$ algebra to justify the EM ansatz. As said above, we have two kinds of Hilbert spaces, one built out of the integral-mode matter oscillators $\alpha_{n}$ in the 25th direction, the other with halfintegral-mode oscillators $J_{r}$, beside all the other matter and ghost oscillators. Let us call them (or, better, their extensions) $\mathcal{H}$ and $\mathcal{H}^{*}$, respectively. The vacuum of the first is the usual string vacuum, made of the tensor product of the various types of oscillators, in particular of the $|0\rangle$ vacuum for the integral oscillators $\alpha_{n}$, with the well-known star product of SFT. The vacuum of the second is obtained by replacing the first vacuum $|0\rangle$ with the state $\left|\sigma_{*}\right\rangle$ (or $\left|\bar{\sigma}_{*}\right\rangle$ ) on which the halfinteger oscillators act. In the first (extended) Hilbert space we have the usual $K, B, c$ algebra and the BRST operator $Q=Q_{\text {ii }}$ acting on it. In the second $K$ is replaced by $K^{*} \equiv$ $K_{\mathrm{ND}} \equiv K_{\mathrm{DN}}$ and $Q$ by $Q^{*} \equiv Q_{\mathrm{ND}} \equiv Q_{\mathrm{DN}}$ and the star product is also modified accordingly.

Let us try to express this in formulas. In the half-integralmode space $\mathcal{H}^{*}$ with one ND or DN direction, the BRST charge is given by

$$
Q^{*}=\sum_{n} c_{n} L_{-n}^{*}+\sum_{m, n} \frac{m-n}{2}: c_{m} c_{n} b_{-m-n}:-c_{0}
$$

where

$$
L_{n}^{*}=\mathcal{L}_{n}+L_{n}^{(d-1)}
$$

where $L_{n}^{(d-1)}$ is the contribution from the NN $X^{i}(i=$ $0, \ldots 24)$ and $\mathcal{L}_{n}$ is as defined above. Instead, for the fully integral-mode space $\mathcal{H}$, the BRST charge is

$$
Q=\sum_{n} c_{n} L_{-n}+\sum_{m, n} \frac{m-n}{2}: c_{m} c_{n} b_{-m-n}:-c_{0} .
$$

We note that, since the Virasoro generators $\mathcal{L}_{n}$ obey the same algebra as $L_{n}$, the proof of the nilpotency of $Q^{*}$ is the same as that of $Q$.

The vacuum state for the half-integral-mode space can be written as

$|0\rangle_{d}=\left|\sigma_{*}\right\rangle \otimes|0\rangle_{d-1} \otimes|0\rangle_{g h}$,

where $\left|\sigma_{*}\right\rangle$ is as defined above and

$$
L_{n}^{(d-1)}|0\rangle_{d-1}=0, \quad \text { for } n \geq-1
$$

$c_{n}|0\rangle_{g h}=0, \quad$ for $n \geq 2, \quad b_{n}|0\rangle_{g h}=0, \quad$ for $n \geq-1$.

We also note that

$\mathcal{L}_{n}\left|\sigma_{*}\right\rangle=0$, for $n \geq 1$

Therefore, the action on the vacuum of the BRST operator $Q^{*}$ is well defined and is given by

$$
\begin{aligned}
Q^{*}|0\rangle_{d}= & \sum_{n} c_{n} \mathcal{L}_{-n}\left|\sigma_{*}\right\rangle \otimes|0\rangle_{d-1} \otimes|0\rangle_{g h} \\
= & \mathcal{L}_{-1}\left|\sigma_{*}\right\rangle \otimes|0\rangle_{d-1} \otimes c_{1}|0\rangle_{g h} \\
& +\mathcal{L}_{0}\left|\sigma_{*}\right\rangle \otimes|0\rangle_{d-1} \otimes c_{0}|0\rangle_{g h} \\
= & \mathcal{L}_{-1}\left|\sigma_{*}\right\rangle \otimes|0\rangle_{d-1} \otimes c_{1}|0\rangle_{g h} \\
& +\frac{1}{16}\left|\sigma_{*}\right\rangle \otimes|0\rangle_{d-1} \otimes c_{0}|0\rangle_{g h}
\end{aligned}
$$

Translated into the BRST variation of the primary operator $\sigma_{*}(s)$, this can be written

$Q^{*} \sigma_{*}(s)=c(s) \partial \sigma_{*}(s)+\frac{1}{16} \partial c(s) \sigma_{*}(s)$.

Now let us introduce $\sigma(s)=\sigma_{*}(s) e^{\frac{i}{4} X^{0}(s)}, \bar{\sigma}(s)=\bar{\sigma}_{*}(s)$ $e^{-\frac{i}{4} X^{0}(s)}$, which are the conformal dimension zero bcc operators used in the EM ansatz, and use the shortcut notation

$\sigma(s)=\sigma_{*}(s) \omega(s), \quad \bar{\sigma}(s)=\bar{\sigma}_{*}(s) \bar{\omega}(s)$.

The corresponding state $|\sigma\rangle$ is defined as follows:

$|\sigma\rangle=\left|\sigma_{*}\right\rangle \otimes|\omega\rangle \otimes|0\rangle_{g h}$

where $|\omega\rangle=\omega(0)|0\rangle_{d-1}$. The variation of this state with respect $Q^{*}$ is

$$
\begin{aligned}
Q^{*}|\sigma\rangle= & \sum_{n} \mathcal{L}_{-n}\left|\sigma_{*}\right\rangle \otimes|\omega\rangle \otimes c_{n}|0\rangle_{g h} \\
& +\sum_{n}\left|\sigma_{*}\right\rangle \otimes L_{-n}^{(0)}|\omega\rangle \otimes c_{n}|0\rangle_{g h} \\
= & \mathcal{L}_{-1}\left|\sigma_{*}\right\rangle \otimes|\omega\rangle \otimes c_{1}|0\rangle_{g h} \\
& +\frac{1}{16}\left|\sigma_{*}\right\rangle \otimes|\omega\rangle \otimes c_{0}|0\rangle_{g h} \\
& +\left|\sigma_{*}\right\rangle \otimes L_{-1}^{(0)}|\omega\rangle \otimes c_{1}|0\rangle_{g h} \\
& -\frac{1}{16}\left|\sigma_{*}\right\rangle \otimes|\omega\rangle \otimes c_{0}|0\rangle_{g h} \\
= & \mathcal{L}_{-1}\left|\sigma_{*}\right\rangle \otimes|\omega\rangle \otimes c_{1}|0\rangle_{g h}+\left|\sigma_{*}\right\rangle \\
& \otimes L_{-1}^{(0)}|\omega\rangle \otimes c_{1}|0\rangle_{g h} .
\end{aligned}
$$

Translated into the BRST variation of the primary operator $\sigma(s)$, this is written as 
$Q^{*} \sigma(s)=c(s) \partial \sigma_{*}(s) \omega(s)+c(s) \sigma_{*}(s) \partial \omega(s)=c(s) \partial \sigma(s)$.

A similar relation also holds for $\bar{\sigma}$.

So the actions of $Q$ and $Q^{*}$ on the relevant vacua are well defined. But it is easy to see that distinguishing between $\mathcal{H}$ and $\mathcal{H}^{*}, K$ and $K^{*}$ is not enough. Expressions like (19) are products of string states belonging to different Hilbert spaces

$\Psi_{\mathrm{NN}} \Psi_{\mathrm{ND}} \Psi_{\mathrm{DD}} \Psi_{\mathrm{DN}} \Psi_{\mathrm{NN}}$

The first, third, and fifth state belong to spaces of type $\mathcal{H}$, the remaining ones to spaces of type $\mathcal{H}_{*}$. But to be accurate we must introduce four Hilbert spaces $\mathcal{H}_{\mathrm{NN}}, \mathcal{H}_{\mathrm{DD}}, \mathcal{H}_{\mathrm{ND}}$, and $\mathcal{H}_{\text {DN }}$. We tensor them with the remaining matter and ghost sectors and give them the same name. The corresponding $Q$ and $K$ will be labeled in the same way, while $c$ and $B$ are universal. In particular $Q_{\mathrm{NN}}$ and $Q_{\mathrm{DD}}$ are (on zero momentum states) of type $Q$, and $Q_{\mathrm{ND}}$ and $Q_{\mathrm{DN}}$ are of type $Q^{*}$.

This poses several problems we have not considered so far.

- What is the star product between string states belonging to different Hilbert spaces?

- The SFT BRST operator $Q=Q_{\mathrm{NN}}$ acts as a derivation on any expression like (57): why is it that it may change by taking the form appropriate to different Hilbert spaces?

The answer to the first question does not seem to be unsurmountable in view of the definition of star product in terms of a three-strings vertex $[40,41]$. The second problem is more complicated. For instance, how is it possible that $Q$ becomes of type $Q^{*}$ whenever it comes across a factor belonging to $\mathcal{H}^{*}$ and returns back to $Q$ if the subsequent factor belongs to $\mathcal{H}$ ? To justify it we have envisaged two possible alternatives.

First alternative. A way to realize this is by introducing intertwining operators between the different Hilbert spaces. Let us call them $X_{\mathrm{NN}, \mathrm{ND}}, X_{\mathrm{ND}, \mathrm{DD}}, X_{\mathrm{DD}, \mathrm{DN}}$, and $X_{\mathrm{DN}, \mathrm{NN}}$ (we think the names are self-explanatory). Next we rewrite (57) as follows:

$\Psi_{\mathrm{NN}} X_{\mathrm{NN}, \mathrm{ND}} \Psi_{\mathrm{ND}} X_{\mathrm{ND}, \mathrm{DD}} \Psi_{\mathrm{DD}} X_{\mathrm{DD}, \mathrm{DN}} \Psi_{\mathrm{DN}} X_{\mathrm{DN}, \mathrm{NN}} \Psi_{\mathrm{NN}}$

and the relation among the different BRST operators is assumed to be

$$
\begin{gathered}
Q_{\mathrm{NN}} X_{\mathrm{NN}, \mathrm{ND}}=X_{\mathrm{NN}, \mathrm{ND}} Q_{\mathrm{ND}}, \\
Q_{\mathrm{ND}} X_{\mathrm{ND}, \mathrm{DD}}=X_{\mathrm{ND}, \mathrm{DD}} Q_{\mathrm{DD}}, \\
Q_{\mathrm{DD}} X_{\mathrm{DD}, \mathrm{DN}}=X_{\mathrm{DD}, \mathrm{DN}} Q_{\mathrm{DN}}, \\
Q_{\mathrm{DN}} X_{\mathrm{DN}, \mathrm{NN}}=X_{\mathrm{DN}, \mathrm{NN}} Q_{\mathrm{NN}} .
\end{gathered}
$$

Since any state $K$ is generated by the corresponding $Q$ acting on $B$ we have also to assume

$$
\begin{gathered}
K_{\mathrm{NN}} X_{\mathrm{NN}, \mathrm{ND}}=X_{\mathrm{NN}, \mathrm{ND}} K_{\mathrm{ND}} \\
K_{\mathrm{ND}} X_{\mathrm{ND}, \mathrm{DD}}=X_{\mathrm{ND}, \mathrm{DD}} K_{\mathrm{DD}} \\
K_{\mathrm{DD}} X_{\mathrm{DD}, \mathrm{DN}}=X_{\mathrm{DD}, \mathrm{DN}} K_{\mathrm{DN}} \\
K_{\mathrm{DN}} X_{\mathrm{DN}, \mathrm{NN}}=X_{\mathrm{DN}, \mathrm{NN}} K_{\mathrm{NN}}
\end{gathered}
$$

If these relations are valid and we have the following modified version of the OPE (14):

$\left(X_{\mathrm{DD}, \mathrm{DN}} \bar{\sigma} X_{\mathrm{DN}, \mathrm{NN}}\right)\left(X_{\mathrm{NN}, \mathrm{ND}} \sigma X_{\mathrm{ND}, \mathrm{DD}}\right)=1$,

some of the issues raised in Sect. 3 are resolved, and we can prove the equation of motion and also compute the energy, provided that the $X$ are pure matter operators (see Appendix A).

Of course all the above works if the intertwining $X$ operators exist. Do they? The possibility of an intertwining operator is envisaged in [31], accompanied by the sentence "The operator so constructed is rather unwieldy". But let us see what these authors refer to. The construction goes back to the 1970s and is due to Corrigan and Fairlie [32], but see also [33-39] for the environment (the search for off-shell dual amplitudes) where such an idea was born. Bcc operators are not mentioned, but they are precisely what Ref. [32] deals with. More precisely that paper is concerned with the relation between what we call $|0\rangle \equiv|0\rangle_{25}$ and $\left|\sigma_{*}\right\rangle$, and it defines an intertwining operator for the vertex operators in the two pictures (integer- and half-integer mode oscillators), say $V(k, z)$ constructed with $\alpha_{n}$ and $\tilde{V}(k, z)$ constructed with the half-mode ones ( $k$ is the momentum). The basic formula is

$$
\begin{aligned}
& \left\langle 0|\exp F(w)| \sigma_{*}\right\rangle V(k, z) \\
& \quad=\lambda e^{-i k \cdot x_{0}} \tilde{V}(k, z-w)\langle 0| \exp F\left(w\left|\sigma_{*}\right\rangle\right.
\end{aligned}
$$

where the quadratic form $F$ is given by

$$
\begin{aligned}
F(z)= & \frac{1}{2} \frac{1}{(2 \pi i)^{2}} \oint_{C} \frac{\mathrm{d} x \mathrm{~d} y}{x y} P(x) A(x, y, z) P(y) \\
& +\frac{1}{(2 \pi i)^{2}} \oint_{C^{\prime}} \frac{\mathrm{d} x \mathrm{~d} y}{x y} P(x) B(x, 1 / y, z) S(y)
\end{aligned}
$$

where $P(z)=-z \frac{d}{\mathrm{~d} z} X_{\mathrm{NN}}(z)$ and $S(z)=-z J_{\mathrm{ND}}(z)$, while

$$
\begin{aligned}
A(x, y, z) & =\sum_{n, m=0}^{\infty} x^{n} A_{n, m}(z) y^{m} \\
& =2 \log (\sqrt{x-z}+\sqrt{y-z}), \\
\lambda & =2^{k^{2}}
\end{aligned}
$$


and

$$
\begin{aligned}
B(x, 1 / y, z) & =\sum_{n=0}^{\infty} \sum_{r=\frac{1}{2}}^{\infty} x^{n} B_{n, r}(z) y^{-r} \\
& =\log \left(\frac{\sqrt{y}-\sqrt{x-z}}{\sqrt{y}+\sqrt{x-z}}\right) .
\end{aligned}
$$

The integration contours must be such that for $C|x|$ and $|y|<|z|$, while for $C^{\prime}|x|<|z|$ but $|y|>|x-z|$. The intertwining operator $\left\langle 0|\exp F(w)| \sigma_{*}\right\rangle$ works very well for vertex operators, but it is easy to see that, unfortunately, it cannot work for expressions like $K_{1}^{L}$ and $Q$. This example gives an explicit idea of what we mean by an intertwining operator in the present context. We do not know whether this construction can be improved, so as to intertwine also $K_{\mathrm{NN}}$ and $Q_{\mathrm{NN}}$ with $K_{\mathrm{ND}}$ and $Q_{\mathrm{ND}}$, and to intertwine the star products in the two sector. We think it should be possible, but our attempts in this direction have failed.

The idea of considering two different Fock spaces $\mathcal{H}$ and $\mathcal{H}^{*}$ with an intertwining operator between them, if viable, would solve the problem. However, such a construction would make explicit use of DD, ND, and DN oscillators, beside the NN ones. The former appear in strings attached to a D24-brane. In fact they define the D24-brane (the dynamics of a brane is defined by the strings attached to it). This means that the information we want the solution to contain, i.e. the description of the D24-brane, is already contained in the initial data. This implies that the solution is background dependent. There is nothing wrong, of course, in trying to describe D-branes in a background dependent way. Background dependence is standard in ordinary string theory approaches, for instance in [29]. But here we are in SFT and, as explained above, the ambition of this theory is background independence, which means that we can derive all the other possible backgrounds as analytic solutions of its equation of motion. It goes without saying that any such solution must be formulated in the original SFT background (the NN string).

This is a good point to recall that the non-analytic or approximate lump solutions one finds in the literature do not make use of bcc operators or half-integer modes. This is the case for numerical solutions based on level truncations, [42-45], but also for the lump solutions in vacuum SFT (see, for instance [46]) and for those in boundary SFT (see, for instance [47]). In analogy we expect that an analytic lump solution should not contain any built-in information about Dbranes, but rather a D-brane description should emerge from the physical content of the solution. A comparison with the BMT proposal below may help to understand the difference.

Second alternative. A radically different and more appealing alternative could be inspired by the example of marginal deformation to the TV solutions. To this end let us recall the KOS solutions [12].

\subsection{The KOS bcc operator}

In the case of the KOS solution for marginal deformations, the bcc operators $\sigma$ and $\bar{\sigma}$ are such that

$\sigma(0) \bar{\sigma}(\alpha)=\exp \left[\int_{0}^{\alpha} \mathrm{d} t V(t)\right]$

where $V$ is a matter primary operator of conformal dimension 1 and it belongs to the original Hilbert space. Therefore, the bcc operators belong to the same Hilbert space. $V$ has the following properties:

$[B, V]=[c, V]=0, \quad Q V=[K, c V]$.

The wedge states with the modified boundary condition are given by

$e^{\alpha(K+V)}=\sigma e^{\alpha K} \bar{\sigma}$.

The BRST variation of the deformed wedge state is

$$
\begin{aligned}
Q e^{\alpha(K+V)} & =\int_{0}^{\alpha} \mathrm{d} t e^{t(K+V)} Q(K+V) e^{(\alpha-t)(K+V)} \\
& =e^{\alpha(K+V)}(c V)-(c V) e^{\alpha(K+V)} .
\end{aligned}
$$

On the other hand using (67) we can write

$$
Q e^{\alpha(K+V)}=(Q \sigma) e^{\alpha K} \bar{\sigma}+\sigma e^{\alpha K}(Q \bar{\sigma}) .
$$

Comparing the last two equations the authors of [12] conclude that

$$
\begin{aligned}
e^{\alpha(K+V)}(c V) & =\sigma e^{\alpha K}(Q \bar{\sigma}), \quad-(c V) e^{\alpha(K+V)} \\
& =(Q \sigma) e^{\alpha K} \bar{\sigma} .
\end{aligned}
$$

Setting $\alpha=0$ and multiplying the first relation by $\bar{\sigma}$ from the left and the second one by $\sigma$ from the right we obtain

$Q \bar{\sigma}=c \bar{\sigma} V, \quad \sigma=-c V \bar{\sigma}$.

We see that the original BRST operator operates effectively on the bcc operators of the KOS solutions, because they belong to the original Hilbert space.

The KOS solutions are based essentially on Eq. (65). Returning to the EM ansatz, the first difference we notice is that in the latter the field analogous to $V$ does not exist (in the relevant case it is singular [18]). Thus we have to proceed in another way. In [27] the half-integral-mode sector is called 'Ramond'. This is an unconventional terminology, but it is reminiscent of the Ramond sector in superstring theories and suggests a parallel with that situation. We recall that in open superstring theory we have two vacua, the NS one, 
analogous to the usual vacuum in bosonic string theory, and the Ramond vacuum (which is made of spinor states forming a representation of the gamma matrix algebra in 10D). It is, however, possible to define a primary operator that, applied to the usual vacuum, creates the Ramond vacuum under the state-operator correspondence. This primary is constructed using (matter and ghost) fields in the theory (with fermion and superghost fields in bosonized form).

Applying this analogy to the EM proposal we may ask whether the $\left|\sigma_{*}\right\rangle$ (and $\left|\bar{\sigma}_{*}\right\rangle$ ) vacuum can be created in an analogous way starting from the ordinary vacuum and applying a primary operator formed with the fields in the theory. In such a case all $Q$ 's and $K$ 's would collapse to the same operator and the previous difficulties would disappear, because there would be no need to distinguish between string states belonging to the four different Hilbert spaces. This scheme is certainly most appealing, in particular it would guarantee background independence. But it is not easy to implement. It cannot be done straight away, because we have at our disposal only the bosonic NN field $X$ (which has no charge at infinity, and thus does not offer any chance to use the Coulomb gas method ${ }^{3}$ ). At page 5 of [28] we find the peremptory sentence: "In open strings there is no such thing as a twisted state", where 'twisted state' refers to $\left|\sigma_{*}\right\rangle$. Perhaps this is too strong a statement, but, certainly, there seems to be no straightforward way to implement this scheme.

In conclusion we have not been able to implement the EM prescriptions in a concrete $2 D$ field theory formalism, consistent with 2D CFT on which SFT is defined, while avoiding background dependence. But, since ours is not a mathematical theorem, we cannot completely exclude that it is possible.

The parallel with the KOS solution suggests one more consideration. When one tries to translate the KOS solution into the EM scheme one comes across a singularity (in the analog of $V$ ). This may be an indication that lump solutions are inevitably, in some sense, singular. A similar peculiarity is met in numerous classical field theory solutions and, in itself, is not really important. What is important in these cases is that the physical quantities related to the solutions can be computed. This is what happens also for the BMT solution.

\section{The BMT proposal}

In [15] a general method has been proposed to obtain new exact analytic solutions in open string field theory, and in particular solutions that describe inhomogeneous tachyon condensation. The method consists in translating an exact renormalization group $(\mathrm{RG})$ flow generated in a two-dimensional world-sheet theory by a relevant operator, into the language of OSFT. The so-constructed solution is a deformation of the

\footnotetext{
${ }^{3}$ Needless to say $e^{\frac{1}{4} X}$, where $X$ is as in (33), is not what we need.
}

ES solution. It has been shown in [15] that, if the operator has suitable properties, the solution will describe tachyon condensation only in specific space directions, thus representing the condensation of a lower dimensional brane. In the following, after describing the general method, we will focus on a particular solution, generated by an exact RG flow first analyzed by Witten [48]. On the basis of the analysis carried out in the framework of 2D CFT in [47], we expect it to describe a D24-brane, with the correct ratio of tension with respect to the starting D25-brane.

Let us see first the general recipe to construct such kind of lump solutions. To start with we enlarge the $K, B, c$ algebra by adding a (relevant) matter string field $\phi$, where

$\phi=\phi\left(\frac{1}{2}\right)|I\rangle, \quad c=c\left(\frac{1}{2}\right)|I\rangle$,

with the properties

$$
\begin{gathered}
{[c, \phi]=0, \quad[B, \phi]=0, \quad[K, \phi]=\partial \phi,} \\
Q \phi=c \partial \phi+\partial c \delta \phi .
\end{gathered}
$$

It can easily be proven that

$\psi_{\phi}=c \phi-\frac{1}{K+\phi}(\phi-\delta \phi) B c \partial c$

does indeed satisfy (in the sense specified below) the OSFT equation of motion

$Q \psi_{\phi}+\psi_{\phi} \psi_{\phi}=0$.

It is clear that (70) is a deformation of the Erler-Schnabl solution, which can be recovered for $\phi=1$.

After some algebraic manipulations one can show that

$\mathcal{Q}_{\psi_{\phi}} \frac{B}{K+\phi}=Q \frac{B}{K+\phi}+\left\{\psi_{\phi}, \frac{B}{K+\phi}\right\}=1$.

So, unless the string field $\frac{B}{K+\phi}$ is singular, it defines a homotopy operator and the solution has trivial cohomology, which is the defining property of the tachyon vacuum [2]. On the other hand, in order for the solution to be well defined, the quantity $\frac{1}{K+\phi}(\phi-\delta \phi)$ should be well defined. Moreover, in order to be able to show that (70) satisfies the equation of motion, one needs $K+\phi$ to be invertible.

In full generality we thus have a new nontrivial solution if, roughly speaking,

1. $\frac{1}{K+\phi}$ is in some sense singular, but

2. the RHS of (70) is regular and

3. $\frac{1}{K+\phi}(K+\phi)=1$.

These conditions seem hard to satisfy and even contradictory. It is indeed so without adequate specifications. This problem was discussed in $[16,54]$, where it was shown that the right framework for a correct interpretation is distribution theory, which guarantees not only regularity of the solution but also 
its 'non-triviality', in the sense that if these conditions are satisfied, it cannot fall in the same class as the ES tachyon vacuum solution. These questions will be discussed further on.

For concreteness the world-sheet RG flow, referred to above, is represented by a parameter $u$, where $u=0$ corresponds to the UV and $u=\infty$ to the IR (in 2D), and $\phi$ is labeled by $\phi_{u}$, with $\phi_{u=0}=0$. Then we require for $\phi_{u}$ the following properties under the coordinate rescaling $f_{t}(z)=\frac{z}{t}$ :

$f_{t} \circ \phi_{u}(z)=\frac{1}{t} \phi_{t u}\left(\frac{z}{t}\right)$.

In [15] a specific relevant operator $\phi_{u}$ and the corresponding SFT solution was considered. This operator generates an exact RG flow and it was studied by Witten in [48], see also [47], and is based on the operator (defined in the cylinder $C_{T}$ of width $T$ in the arctan frame)

$\phi_{u}(s)=u\left(X^{2}(s)+2 \ln u+2 A\right)$

where $X=X^{25}$ is the NN open string field and $A$ is a constant. On the unit disk $D$ we have

$\phi_{u}(\theta)=u\left(X^{2}(\theta)+2 \ln \frac{T u}{2 \pi}+2 A\right)$.

If we set

$g_{A}(u)=\left\langle e^{-\frac{1}{2 \pi} \int_{0}^{4 \pi} \mathrm{d} \theta u\left(X^{2}(\theta)+2 \ln \frac{u}{2 \pi}+2 A\right)}\right\rangle_{D}$

we get

$g_{A}(u)=Z(2 u) e^{-2 u\left(\ln \frac{u}{2 \pi}+A\right)}$

where $Z(u)$ is the partition function of the system on the unit disk computed by [48]. Requiring finiteness for $u \rightarrow \infty$ one gets $A=\gamma-1+\ln 4 \pi$, which implies

$$
\begin{aligned}
g_{A}(u) \equiv g(u) & =\frac{1}{2 \sqrt{\pi}} \sqrt{2 u} \Gamma(2 u) e^{2 u(1-\ln (2 u))}, \\
\lim _{u \rightarrow \infty} g(u) & =1 .
\end{aligned}
$$

Moreover, as it turns out, $\phi_{u}-\delta \phi_{u}=u \partial_{u} \phi_{u}(s)$.

The $\phi_{u}$ just introduced satisfies all the requested properties. According to [47], the corresponding RG flow in BCFT reproduces the correct ratio of tension between D25- and D24-branes. Consequently $\psi_{u} \equiv \psi_{\phi_{u}}$ is expected to represent a D24-brane solution.

In SFT the most important gauge invariant quantity is of course the energy. Therefore in order to make sure that $\psi_{u} \equiv$ $\psi_{\phi_{u}}$ is the expected solution we must prove that its energy equals a D24-brane energy.

The energy expression for the lump solution was determined in [15] by evaluating a three-point function on the cylinder $C_{T}$. It equals $-\frac{1}{6}$ times the following expression:

$$
\begin{aligned}
& \left\langle\psi_{u} \psi_{u} \psi_{u}\right\rangle=-\int_{0}^{\infty} \mathrm{d} t_{1} \mathrm{~d} t_{2} \mathrm{~d} t_{3} \mathcal{E}_{0}\left(t_{1}, t_{2}, t_{3}\right) u^{3} g(u T) \\
& \quad \times\left\{\left(-\frac{\partial_{2 u T} g(u T)}{g(u T)}\right)^{3}+\frac{1}{2}\left(-\frac{\partial_{2 u T} g(u T)}{g(u T)}\right)\right. \\
& \times\left(G_{2 u T}^{2}\left(\frac{2 \pi t_{1}}{T}\right)+G_{2 u T}^{2}\left(\frac{2 \pi\left(t_{1}+t_{2}\right)}{T}\right)\right. \\
& \left.+G_{2 u T}^{2}\left(\frac{2 \pi t_{2}}{T}\right)\right) \\
& \left.+G_{2 u T}\left(\frac{2 \pi t_{1}}{T}\right) G_{2 u T}\left(\frac{2 \pi\left(t_{1}+t_{2}\right)}{T}\right) G_{2 u T}\left(\frac{2 \pi t_{2}}{T}\right)\right\} .
\end{aligned}
$$

Here $T=t_{1}+t_{2}+t_{3}$ and $g(u)$ is as above, while $G_{u}(\theta)$ represents the boundary-to-boundary correlator first determined by Witten [48]:

$G_{u}(\theta)=\frac{1}{u}+2 \sum_{k=1}^{\infty} \frac{\cos (k \theta)}{k+u}$.

Finally, $\mathcal{E}_{0}\left(t_{1}, t_{2}, t_{3}\right)$ represents the ghost three-point function in $C_{T}$,

$$
\begin{aligned}
\mathcal{E}_{0}\left(t_{1}, t_{2}, t_{3}\right) & =\left\langle B c \partial c\left(t_{1}+t_{2}\right) \partial c\left(t_{1}\right) \partial c(0)\right\rangle_{C_{T}} \\
& =-\frac{4}{\pi} \sin \frac{\pi t_{1}}{T} \sin \frac{\pi\left(t_{1}+t_{2}\right)}{T} \sin \frac{\pi t_{2}}{T} .
\end{aligned}
$$

A remarkable property of (77) is that it does not depend on $u$. In fact $u$ can be absorbed in a redefinition of variables $t_{i} \rightarrow u t_{i}, i=1,2,3$, and it disappears from the expression.

The integral in (77) is well defined in the IR ( $s$ very large, setting $s=2 u T)$ but has a UV $(s \approx 0)$ singularity, which must be subtracted away. ${ }^{4}$ Once this is done, Eq. (77) can be numerically computed, the result being $\approx 0.069$. This is not the expected result, but this is not surprising, for the result depends on the UV subtraction. Therefore one cannot assign to it any physical significance. To get a meaningful result we must return to the very meaning of Sen's third conjecture, which says that the lump solution is a solution of the theory on the tachyon condensation vacuum. Therefore we must measure the energy of our solution with respect to the tachyon condensation vacuum. Simultaneously the resulting energy must be a subtraction-independent quantity because only to such a quantity can a physical meaning be assigned. Both requirements have been satisfied in [16] in the following way.

First a new solution to the EOM, depending on a parameter $\varepsilon$, has been introduced

$\psi_{u}^{\varepsilon}=c\left(\phi_{u}+\varepsilon\right)-\frac{1}{K+\phi_{u}+\varepsilon}\left(\phi_{u}+\varepsilon-\delta \phi_{u}\right) B c \partial c$

\footnotetext{
${ }^{4}$ Of course this singularity is present also in the ES solution, it is represented by the infinite volume. The difference here is that the infinite volume appears in the form of a zero mode which generates a singularity $\sim \frac{1}{\sqrt{u}}$ in $g(u)$.
} 
in the limit $\varepsilon \rightarrow 0$. This limit will be mostly understood from now on. The energy of (78) (after the same UV subtraction as in the previous case $\mathrm{e}^{5}$ ) is (numerically) 0 . Since (unlike the previous case) the presence of the parameter $\varepsilon$ prevents the IR transition to a new critical point, it seems sensible to assume that $\lim _{\varepsilon \rightarrow 0} \psi_{u}^{\varepsilon}$ represents the tachyon condensation vacuum solution. In other words we assume it is gauge equivalent to the ES solution (we will justify this further on). Using it, a solution to the EOM at the tachyon condensation vacuum has been obtained. The equation of motion at the tachyon vacuum is

$\mathcal{Q} \Phi+\Phi \Phi=0$, where $\mathcal{Q} \Phi=Q \Phi+\psi_{u}^{\varepsilon} \Phi+\Phi \psi_{u}^{\varepsilon}$

One can easily show that

$\Phi_{0}=\psi_{u}-\psi_{u}^{\varepsilon}$

is a solution to (79). The action at the tachyon vacuum is $-\frac{1}{2}\langle\mathcal{Q} \Phi, \Phi\rangle-\frac{1}{3}\langle\Phi, \Phi \Phi\rangle$. Thus the energy of $\Phi_{0}$ is

$$
\begin{aligned}
E\left[\Phi_{0}\right]= & -\frac{1}{6}\left\langle\Phi_{0}, \Phi_{0} \Phi_{0}\right\rangle \\
= & -\frac{1}{6}\left[\left\langle\psi_{u}, \psi_{u} \psi_{u}\right\rangle-\left\langle\psi_{u}^{\varepsilon}, \psi_{u}^{\varepsilon} \psi_{u}^{\varepsilon}\right\rangle\right. \\
& \left.-3\left\langle\psi_{u}^{\varepsilon}, \psi_{u} \psi_{u}\right\rangle+3\left\langle\psi_{u}, \psi_{u}^{\varepsilon} \psi_{u}^{\varepsilon}\right\rangle\right] .
\end{aligned}
$$

The UV subtractions necessary for each correlator at the RHS of this equation are the same in all cases, therefore they cancel out and the final result is subtraction-independent. A final bonus of this procedure is that the final result can be derived purely analytically and $E\left[\Phi_{0}\right]$ turns out to be precisely the D24-brane energy. With the conventions of [16], this is

$T_{D 24}=\frac{1}{2 \pi^{2}}$

In [17] the same result was extended to Dp-brane lump solutions for any $p$. All these solutions are background independent.

Before we pass to a closer scrutiny of this solution and its properties, we need a discussion of the relations between various solutions of the SFT EoM provided by gauge transformations. What we would like to stress is that gauge equivalent solutions may take very different (even singular) forms. To this end we make a detour about identity based (IB) solutions.

\footnotetext{
5 The parameter $\varepsilon$ is originally a gauge parameter, but due to the UV subtraction such a gauge nature is broken and the energy functional depends softly on the value of $\varepsilon$ [49].
}

\section{A detour: TV solution as gauge transformation of IB solutions}

Let us introduce the following family of IB solutions [50-53], depending on a parameter $\alpha$ :

$\psi_{\alpha}=c(\alpha-K)$

which can also be written as a pure gauge solution as follows:

$\psi_{\alpha}=U_{\alpha}^{-1} Q U_{\alpha}$

where

$U_{\alpha}=1-\frac{1}{\alpha} c B(\alpha-K), \quad U_{\alpha}^{-1}=1+c B \frac{\alpha-K}{K}$.

We note that for the $\alpha=0$ case the gauge transformation is singular. The homotopy field is $\mathcal{A}_{\alpha}=\frac{1}{\alpha} B$. The problem with IB solutions is in the computation of the physical observable. For example, the direct evaluation of the energy using the $K, B, c$ algebra gives zero:

$$
\begin{aligned}
E \sim\left\langle\psi_{\alpha}^{3}\right\rangle & =-\langle c K c K c K\rangle \\
& =-\langle c K c \partial c K\rangle=-\left\langle c K(\partial c)^{2} K\right\rangle=0 .
\end{aligned}
$$

However, if we use a different regularization method we will get different results. The most obvious regularization is the following:

$$
\begin{aligned}
\left\langle\psi_{\alpha}^{3}\right\rangle & =-\lim _{t_{i} \rightarrow 0}\left\langle c K \Omega^{t_{1}} c K \Omega^{t_{2}} c K \Omega^{t_{3}}\right\rangle \\
& =\lim _{t_{i} \rightarrow 0} \partial_{t_{1}} \partial_{t_{2}} \partial_{t_{3}}\left\langle c \Omega^{t_{1}} c \Omega^{t_{2}} c \Omega^{t_{3}}\right\rangle
\end{aligned}
$$

The answer in this case depends on the way we take the limits, therefore this regularization is ambiguous. Another procedure is to connect this solution to the class of ES TV solutions by a regular gauge transformation [51-53]. This way we generate from the IB solutions the following two parameters family of solutions:

$$
\begin{aligned}
\Psi_{\alpha, \lambda} & =V_{\lambda}^{-1}\left(\psi_{\alpha}+Q\right) V_{\lambda} \\
& =\frac{1}{\lambda} c(1+\lambda K B c)\left(\frac{1}{1+\lambda K}+\lambda \alpha-1\right)
\end{aligned}
$$

where

$V_{\lambda}^{-1}=1+\lambda c B K, \quad V_{\lambda}=1-\lambda c B K \frac{1}{1+\lambda K}$.

If we set $\lambda=\alpha=1$, we obtain the ES TV solution. Since all the known solutions can formally be written as a pure gauge, for any solution $\Psi=U_{\Psi}^{-1} Q U_{\Psi}$ we can write

$$
\begin{aligned}
\Psi & \equiv U_{\Psi}^{-1} Q U_{\Psi} \equiv V_{\Psi}^{-1}\left(\psi_{\alpha}+Q\right) V_{\Psi} \\
& =V_{\Psi}^{-1}\left(U_{\alpha}^{-1} Q U_{\alpha}+Q\right) V_{\Psi},
\end{aligned}
$$

where

$$
V_{\Psi}=U_{\alpha}^{-1} U_{\Psi}
$$


For the TV solutions this gauge transformation is regular, which means they are gauge equivalent to the IB solutions.

The energy for these class of solutions in (87) is independent of both $\alpha$ and $\lambda$ and it is equal to the energy of the ES TV solution. The same is true for the closed string overlap. Since the original Schnabl's TV solution is also gauge equivalent to the ES solution, we see that all the known TV solutions are regular gauge transforms of the IB solutions.

What is interesting about the generalized TV solution $\Psi_{\alpha, \lambda}$ is related to its homotopy field. The homotopy field is given by

$\mathcal{A}_{\alpha, \lambda}=V_{\lambda}^{-1} \mathcal{A}_{\alpha} V_{\lambda}=\frac{1}{\alpha} \frac{B}{1+\lambda K}$,

which is a well-defined field for $\alpha \neq 0$ and $\lambda \geq 0$. However, the solution itself and the corresponding physical observables are well defined for $\alpha=0$ as well. We cannot tell if the cohomology of the corresponding BRST operator is vanishing for $\alpha=0$. We know that the existence of the homotopy field is a sufficient condition for the vanishing of the cohomology, but is it a necessary condition? We will return to this point later on.

7.1 BMT lump solution as gauge transformation of IB solutions

Let us apply the previous formalism to the BMT lump solution. In particular we write the gauge transformation which relates the BMT lump solution to the identity based solution. Since the parameter $\lambda$ has no significance we set it to 1 ,

$\Psi_{\phi, \alpha} \equiv U_{\phi}^{-1} Q U_{\phi} \equiv V_{\phi}^{-1}\left(\psi_{\alpha}+Q\right) V_{\phi}$,

where $V_{\phi}=U_{\alpha}^{-1} U_{\phi}$ is given by

$$
\begin{aligned}
& V_{\phi}^{-1}=1+c B(K+\phi-1), \\
& V_{\phi}=1-c B\left(1-\frac{1}{K+\phi}\right) .
\end{aligned}
$$

After some simplifications we obtain

$$
\Psi_{\phi, \alpha}=\alpha \phi c+(\alpha-1) c K B c-c K c B(\phi-\delta \phi) \frac{1}{K+\phi} .
$$

We note that for $\alpha=1$ this gives the $\psi_{\phi}$ BMT lump ansatz. The new thing here is that this time the gauge transformation is not regular (see below for an additional comment on regular and singular gauge transformations). Therefore, we can claim that the lump solution is a genuine new solution. The calculation of a physical observable will follow the standard procedure. For example the first two pieces in (93) do not contribute to the energy, so that the energy is $\alpha$ independent and the same as that of the BMT lump solution. Let us focus on the homotopy field. It is given by
$\mathcal{A}_{\phi, \alpha}=V_{\phi}^{-1} \mathcal{A}_{\alpha} V_{\phi}=\frac{1}{\alpha} \frac{B}{\phi+K}$,

which is singular even for $\alpha \neq 0$.

In [16] another regularized solution was used (see above). It was obtained via the replacement $\phi \rightarrow \phi+\varepsilon$. Replacing this in the above formulas gives

$V_{\phi}=1+c B(K+\phi+\varepsilon-1)$,

$V_{\phi}^{-1}=1-c B\left(1-\frac{1}{K+\phi+\varepsilon}\right)$,

and the solution becomes

$$
\begin{aligned}
\Psi_{\phi, \alpha, \varepsilon}= & \alpha(\phi+\varepsilon) c+(\alpha-1) c K B c \\
& -c K c B(\phi+\varepsilon-\delta \phi) \frac{1}{K+\phi+\varepsilon} .
\end{aligned}
$$

The regularized solution is obtained with $\alpha=1$. In [16] it was identified with the TV solution (see also the previous section). The gauge transformation this time is regular because of the $\varepsilon$ parameter, and the solution will reproduce the TV observables. In this case the homotopy field is

$\mathcal{A}_{\phi, \alpha}=\frac{1}{\alpha} \frac{B}{K+\phi+\varepsilon}$,

which is regular as expected (see further comments below on this issue). The regularized solution is also gauge equivalent to the ES solution. Setting $\alpha=1$ we can write

$\Psi_{\phi, \varepsilon}=X^{-1}\left(\Psi_{E S}+Q\right) X$

where

$X^{-1}=1-c B(1-\phi-\varepsilon) \frac{1}{K+1}$,

$X=1+c B(1-\phi-\varepsilon) \frac{1}{K+\phi+\varepsilon}$,

so it has precisely the same energy as the ES solution.

\section{Discussion of BMT solution}

This section is devoted to discussing the critical aspects of the BMT proposal. Let us start with the conditions 1, 2, and 3 of Sect. 6. We have already anticipated there that these, seemingly contradictory, requirements can be satisfied with an appropriate mathematical interpretation. String states such as $\frac{1}{K+\phi}$ or, similarly, $\frac{1}{K}$ may be singular. This is so because the operators that act on $|I\rangle$ in the definition of both are expected to have a nontrivial kernel. In such a case the Schwinger representations

$\frac{1}{K}=\int_{0}^{\infty} \mathrm{d} t e^{-K t}, \quad \frac{1}{K+\phi}=\int_{0}^{\infty} \mathrm{d} t e^{-(K+\phi) t}$

are bound to diverge, due the zero modes of the corresponding operators. To obtain well-behaved Schwinger representations we have to find a way to remove such singularities. 
As was repeatedly noticed in $[16,17,54]$, this is analogous to the procedure of removing singularities, or defining distributions, in ordinary function theory. In [54] a first attempt was made to do the same for $\frac{1}{K+\phi}$. A space $\mathcal{F}$ of test string states or regular states with a definite topology was introduced. The dual of it, $\mathcal{F}^{\prime}$, with the appropriate topology was defined. The state $\frac{1}{K+\phi}$ belongs to the latter, i.e. it is a distribution. As such the condition 2 and 3 of Sect. 6 are satisfied and the SFT equation of motion is verified (on the other hand, in a common-sense approach, a simple continuity argument would be enough to prove the latter; see [16]). Moreover, as was shown above, the procedure to compute the energy is well defined.

As for point 1 of Sect. 6 it is self-evident, but a comment is in order. The string state $\frac{1}{K+\phi}$ must be singular so that the would-be homotopy operator $\frac{B}{K+\phi}$ (understood as the starmultiplication operator by the string state $\frac{B}{K+\phi}$ ) is not well defined, otherwise the perturbative spectrum on the brane would be trivial. A true homotopy operator is supposed to map normalized states (annihilated by the BRST operator) into normalized states. The state $\frac{1}{K+\phi}$ is singular and it has to be regularized. As was shown in [54] this can be done in a weak sense (in physical language, for correlators), while the previous requirement would require an operator topology argument, which does not seem to exist. For this reason we conclude that $\frac{B}{K+\phi}$ does not exist as a homotopy operator.

Let us illustrate this with an example. In the concrete BMT solution in Sect. 7, defined by the relevant perturbation (73), the inhomogeneous tachyon condensation takes place in the limit $u \rightarrow \infty$. Now it is easy to see that

$\mathcal{Q}\left(c \phi_{u}\right)=c K \frac{1}{K+\phi_{u}}\left(\phi_{u}-2 u\right) B c \partial c$

and, naively,

$\lim _{u \rightarrow \infty} \frac{1}{K+\phi_{u}}\left(\phi_{u}-2 u\right)=1$.

Therefore $c \frac{\phi_{u}}{u}$ is a closed state for $\mathcal{Q}$ in the limit $u \rightarrow \infty$, but it is infinite due to the presence of $\log u$ in $\phi_{u}$. On the other hand one can easily show in the same way that $\lim _{u \rightarrow \infty} \mathcal{Q} c=$ 0 . So the (non-singular) state

$\phi^{(a s)}=\lim _{u \rightarrow \infty} c\left(\frac{\phi_{u}}{u}-2 u \log u\right)$

is annihilated by $\mathcal{Q}$ (at least to this naive level of manipulation). Therefore, provided it is nontrivial, it is a candidate to represent a (zero momentum) state in the spectrum of the D24-brane. Now, while it is not hard to imagine a procedure to define a norm for this state, it is impossible to do the same for the state $\frac{B}{K+\phi} \phi^{(a s)}$.

The previous conclusions have important consequences for the themes discussed in this paper. For instance, it is debated what the allowed gauge transformations are in SFT.
Based on the above we propose the following distinction: a gauge transformation is allowed if it is regular in the above sense, that is, if it does not need to be regularized; otherwise it is not allowed. In other words, an allowed gauge transformation cannot be a true distribution. Once this is established, we can return to the end of the previous section: the state $\frac{1}{K+\phi+\varepsilon}$ for $\varepsilon \neq 0$ is not a distribution (it does not need a regularization), it is a regular state. So the states $X$ and $X^{-1}$, which lead from the ES solution to the $\Psi_{\phi, \varepsilon}$ solution (98), (99), are regular. Therefore the corresponding gauge transformation is a genuine one, and the $\Psi_{\phi, \varepsilon}$ solution is equivalent to the ES one. This implies in particular that they have the same energy.

We can now reconsider one of the unsatisfactory aspects of the energy calculation in [16]. As pointed out above, the energy of (96) was calculated only numerically and turned out to be 0 . It was remarked in [16] and repeated above that this value is only conventional, because it is obtained via a UV subtraction which introduces an arbitrariness in the absolute result. Consequently it was stressed in [16] that only quantities that are independent of such subtractions can be assigned a physical meaning, and it was precisely in this way that the lump energy was calculated. From what we have just argued (see also the end of Sect. 7.1) we are now able not only to confirm all this, but also that the energy of $\Psi_{\phi, \varepsilon}$ is precisely the TV energy, thus confirming the intuition in [16].

\subsection{A comment about homotopy operators}

In the previous section we have presented a family of analytic solutions of the OSFT EoM that are gauge equivalent to identity based solutions. They split into two subsets: $\alpha \neq 0$ and $\alpha=0$. The first set is gauge equivalent to the ES solution and has a regular homotopy operator. The second set, although modeled on the ES solution, although regular and with the same energy as the ES solution, is in no obvious way gauge equivalent to it. The latter case is puzzling because, in addition, it does not admit a homotopy operator as a function of $K, B, c$. We recall again that the existence of a homotopy operator, in correspondence with a tachyon condensation vacuum solution $\psi_{0}$, implies that the vacuum has a trivial cohomology, i.e. no perturbative open string spectrum; for $\mathcal{Q}_{\psi_{0}} \phi=0$ implies

$$
\begin{aligned}
\phi & =\left(\mathcal{Q}_{\psi_{0}} \mathcal{A}\right) \phi=\mathcal{Q}_{\psi_{0}}(\mathcal{A} \phi)-\mathcal{A}\left(\mathcal{Q}_{\psi_{0}} \phi\right) \\
& =\mathcal{Q}_{\psi_{0}} \chi, \quad \chi=\mathcal{A} \phi .
\end{aligned}
$$

This condition is also necessary: if any state annihilated by $\mathcal{Q}_{\psi_{0}}$ is trivial, i.e. if $\mathcal{Q}_{\psi_{0}} \phi=0$ implies that $\phi=\mathcal{Q}_{\psi_{0}} \chi$ for some state $\chi$, it means that there exists a map whose domain is the kernel of $\mathcal{Q}_{\psi_{0}}$ and whose image lies in the complement 
of the kernel. This map is linear and is precisely the homotopy operator. $^{6}$

This is puzzling and we face two possibilities: (1) either $\psi_{\alpha=0}$ is truly not equivalent to the ES solution though degenerate in energy with it, in which case it may well be that the perturbative spectrum supported by this solution is not empty; but the physical interpretation would be obscure: what is this vacuum degenerate with the TV supposed to be? or (2) $\psi_{\alpha=0}$ is truly equivalent in some non-evident way to the ES solution, but in this case a homotopy operator should exist. Then we are again faced with two possibilities. The first possibility is that the homotopy operator for $\psi_{\alpha=0}$ exists, but cannot be expressed as a function $K, B, c$. Although unlikely, because the solution $\psi_{\alpha=0}$ is a simple function of $K, B, c$, we cannot completely exclude this exotic possibility. A second, more likely, alternative is that the homotopy operator exists as a limit of a sequence of homotopy operators of analogous solutions. This of course requires the existence of a topology in the space of solutions, that is, in the space of string states. Such a topology should not be confused with the Hilbert space norm, because most of the string states that enter this game have infinite Hilbert space norm. An attempt in this direction has been initiated in [54].

\section{Conclusions}

In this paper we have pointed out the problems connected with the proposal of [18] to construct analytic lump solutions in OSFT. We have remarked that for it to become effective (in a background independent way) one must show that it is possible to implement it in the very same language (2D CFT) in which OSFT is formulated. We have made some attempts in this direction without succeeding. However, we do not completely exclude that such an implementation be possible. We have also made some additional improving remarks on the BMT proposal for analytic lump solutions. This proposal too has a problem of a different nature related to its mathematical background: a formulation of a well-defined distribution theory for string fields that generalizes the approach of [54]. We hope anyhow we have at least brought enough evidence, with this and other examples in Sect. 8.1, that it is time to face the problem of the topology in the space of string fields.

Acknowledgments We would like to thank Carlo Maccaferri for explaining to us his work prior to publication and for other comments, and Ilmar Gaharamanov for collaboration in an early stage of

\footnotetext{
${ }^{6}$ We do not say anything about the properties of the homotopy operator, for instance if it is continuous. To this end one should introduce a topology in the space of string states. This problem is usually ignored in the existing literature, although it is very likely at the origin of many ambiguities that arise in the search for analytic solutions in SFT.
}

the research. The work of D.D.T. was supported by the Grant MIUR $2010 Y J 2 N Y W_{0} 01$.

Open Access This article is distributed under the terms of the Creative Commons Attribution 4.0 International License (http://creativecomm ons.org/licenses/by/4.0/), which permits unrestricted use, distribution, and reproduction in any medium, provided you give appropriate credit to the original author(s) and the source, provide a link to the Creative Commons license, and indicate if changes were made.

Funded by $\mathrm{SCOAP}^{3}$.

\section{Appendix A: EoM and energy with intertwining opera- tors}

In this appendix we verify the equation of motion and energy of the EM solution, rewritten in terms of intertwining operators as in Sect. 5. Equation (16) can be written

$$
\begin{aligned}
\Phi_{0}= & -\frac{1}{\sqrt{1+K_{\mathrm{NN}}}} c\left(1+K_{\mathrm{NN}}\right) X_{1} \sigma X_{2} \\
& \times \frac{B}{1+K_{\mathrm{DD}}} X_{3} \bar{\sigma} X_{4}\left(1+K_{\mathrm{NN}}\right) c \frac{1}{\sqrt{1+K_{\mathrm{NN}}}},
\end{aligned}
$$

where we used $X_{1}=X_{\mathrm{NN}, \mathrm{ND}}, X_{2}=X_{\mathrm{ND}, \mathrm{DD}}, X_{3}=$ $X_{\mathrm{DD}, \mathrm{DN}}$, and $X_{4}=X_{\mathrm{DN}, \mathrm{NN}}$ for simplicity. Using (59), the BRST variation of $\Phi_{0}$ is written as

$$
\begin{aligned}
Q_{\mathrm{NN}} \Phi_{0}= & -\frac{1}{\sqrt{1+K_{\mathrm{NN}}}}\left(Q_{\mathrm{NN}} c\right)\left(1+K_{\mathrm{NN}}\right) \\
& X_{1} \sigma X_{2} \frac{B}{1+K_{\mathrm{DD}}} \\
& \times X_{3} \bar{\sigma} X_{4}\left(1+K_{\mathrm{NN}}\right) c \frac{1}{\sqrt{1+K_{\mathrm{NN}}}} \\
& +\frac{1}{\sqrt{1+K_{\mathrm{NN}}}} c\left(1+K_{\mathrm{NN}}\right) \\
& X_{1}\left(Q_{\mathrm{ND}} \sigma\right) X_{2} \frac{B}{1+K_{\mathrm{DD}}} \\
& \times X_{3} \bar{\sigma} X_{4}\left(1+K_{\mathrm{NN}}\right) c \frac{1}{\sqrt{1+K_{\mathrm{NN}}}} \\
& +\frac{1}{\sqrt{1+K_{\mathrm{NN}}}} c\left(1+K_{\mathrm{NN}}\right) X_{1} \sigma X_{2} \frac{\left(Q_{\mathrm{DD}} B\right)}{1+K_{\mathrm{DD}}} \\
& \times X_{3} \bar{\sigma} X_{4}\left(1+K_{\mathrm{NN}}\right) c \frac{1}{\sqrt{1+K_{\mathrm{NN}}}} \\
& -\frac{1}{\sqrt{1+K_{\mathrm{NN}}}} c\left(1+K_{\mathrm{NN}}\right) X_{1} \sigma X_{2} \frac{B}{1+K_{\mathrm{DD}}} \\
& \times X_{3}\left(Q_{\mathrm{DN}} \bar{\sigma}\right) X_{4}\left(1+K_{\mathrm{NN}}\right) c \frac{1}{\sqrt{1+K_{\mathrm{NN}}}} \\
& -\frac{1}{\sqrt{1+K_{\mathrm{NN}}}} c\left(1+K_{\mathrm{NN}}\right) X_{1} \sigma X_{3} \frac{B}{1+K_{\mathrm{DD}}} \\
& \times X_{3} \bar{\sigma} X_{4}\left(1+K_{\mathrm{NN}}\right)\left(Q_{\mathrm{NN}} c\right) \frac{1}{\sqrt{1+K_{\mathrm{NN}}}} .
\end{aligned}
$$

Unlike what we see in Eq. (31), now the switching of the BRST charge from one Hilbert space to the other when it 
acts on different components of the solution is justified by

(59). Using the $K, B, c, \sigma, \bar{\sigma}$ algebra of Sect. 3 we can write

$$
\begin{aligned}
Q_{\mathrm{NN}} \Phi_{0}= & -\frac{1}{\sqrt{1+K_{\mathrm{NN}}}} c K_{\mathrm{NN}} c(1 \\
& \left.+K_{\mathrm{NN}}\right) X_{1} \sigma X_{2} \frac{B}{1+K_{\mathrm{DD}}} \\
& \times X_{3} \bar{\sigma} X_{4}\left(1+K_{\mathrm{NN}}\right) c \frac{1}{\sqrt{1+K_{\mathrm{NN}}}} \\
& +\frac{1}{\sqrt{1+K_{\mathrm{NN}}}} c\left(1+K_{\mathrm{NN}}\right) c X_{1}\left(K_{\mathrm{ND}} \sigma-\right. \\
& \left.\sigma K_{\mathrm{ND}}\right) X_{2} \frac{B}{1+K_{\mathrm{DD}}} \\
& \times X_{3} \bar{\sigma} X_{4}\left(1+K_{\mathrm{NN}}\right) c \frac{1}{\sqrt{1+K_{\mathrm{NN}}}} \\
& +\frac{1}{\sqrt{1+K_{\mathrm{NN}}}} c\left(1+K_{\mathrm{NN}}\right) X_{1} \sigma X_{2} \frac{K_{\mathrm{DD}}}{1+K_{\mathrm{DD}}} \\
& \times X_{3} \bar{\sigma} X_{4}\left(1+K_{\mathrm{NN}}\right) c \frac{1}{\sqrt{1+K_{\mathrm{NN}}}} \\
& -\frac{1}{\sqrt{1+K_{\mathrm{NN}}}} c\left(1+K_{\mathrm{NN}}\right) X_{1} \sigma X_{2} \frac{B}{1+K_{\mathrm{DD}}} \\
& \times X_{3}\left(K_{\mathrm{DN}} \bar{\sigma}-K_{\mathrm{DN}} \bar{\sigma}\right) X_{4} c(1 \\
& \left.+K_{\mathrm{NN}}\right) c \frac{1}{\sqrt{1+K_{\mathrm{NN}}}} \\
& -\frac{1}{\sqrt{1+K_{\mathrm{NN}}}} c\left(1+K_{\mathrm{NN}}\right) X_{1} \sigma X_{3} \frac{B}{1+K_{\mathrm{DD}}} \\
& \times X_{3} \bar{\sigma} X_{4}\left(1+K_{\mathrm{NN}}\right) c K_{\mathrm{NN}} c \frac{1}{\sqrt{1+K_{\mathrm{NN}}}},
\end{aligned}
$$

where we have used the assumption that the $X_{i} s$ are pure matter operators. Now with the help of (60), we can convert the $K_{i j}$ to $K_{i i}$ to obtain

$$
\begin{aligned}
& Q_{\mathrm{NN}} \Phi_{0}=-\frac{1}{\sqrt{1+K_{\mathrm{NN}}}} c K_{\mathrm{NN}} c\left(1+K_{\mathrm{NN}}\right) X_{1} \sigma X_{2} \frac{B}{1+K_{\mathrm{DD}}} \\
& \times X_{3} \bar{\sigma} X_{4}\left(1+K_{\mathrm{NN}}\right) c \frac{1}{\sqrt{1+K_{\mathrm{NN}}}} \\
& +\frac{1}{\sqrt{1+K_{\mathrm{NN}}}} c\left(1+K_{\mathrm{NN}}\right) c K_{\mathrm{NN}} X_{1} \sigma X_{2} \frac{B}{1+K_{\mathrm{DD}}} \\
& \times X_{3} \bar{\sigma} X_{4}\left(1+K_{\mathrm{NN}}\right) c \frac{1}{\sqrt{1+K_{\mathrm{NN}}}} \\
& -\frac{1}{\sqrt{1+K_{\mathrm{NN}}}} c\left(1+K_{\mathrm{NN}}\right) c X_{1} \sigma X_{2} K_{\mathrm{DD}} \frac{B}{1+K_{\mathrm{DD}}} \\
& \times X_{3} \bar{\sigma} X_{4}\left(1+K_{\mathrm{NN}}\right) c \frac{1}{\sqrt{1+K_{\mathrm{NN}}}} \\
& +\frac{1}{\sqrt{1+K_{\mathrm{NN}}}} c\left(1+K_{\mathrm{NN}}\right) X_{1} \sigma X_{2} \frac{K_{\mathrm{DD}}}{1+K_{\mathrm{DD}}} \\
& \times X_{3} \bar{\sigma} X_{4}\left(1+K_{\mathrm{NN}}\right) c \frac{1}{\sqrt{1+K_{\mathrm{NN}}}}
\end{aligned}
$$

$$
\begin{aligned}
& -\frac{1}{\sqrt{1+K_{\mathrm{NN}}}} c\left(1+K_{\mathrm{NN}}\right) X_{1} \sigma X_{2} \frac{B}{1+K_{\mathrm{DD}}} K_{\mathrm{DD}} \\
& \times X_{3} \bar{\sigma} X_{4} c\left(1+K_{\mathrm{NN}}\right) c \frac{1}{\sqrt{1+K_{\mathrm{NN}}}} \\
& +\frac{1}{\sqrt{1+K_{\mathrm{NN}}}} c\left(1+K_{\mathrm{NN}}\right) X_{1} \sigma X_{2} \frac{B}{1+K_{\mathrm{DD}}} \\
& \times X_{3} \bar{\sigma} X_{4} K_{\mathrm{NN}} c\left(1+K_{\mathrm{NN}}\right) c \frac{1}{\sqrt{1+K_{\mathrm{NN}}}} \\
& -\frac{1}{\sqrt{1+K_{\mathrm{NN}}}} c\left(1+K_{\mathrm{NN}}\right) X_{1} \sigma X_{3} \frac{B}{1+K_{\mathrm{DD}}} \\
& \times X_{3} \bar{\sigma} X_{4}\left(1+K_{\mathrm{NN}}\right) c K_{\mathrm{NN}} c \frac{1}{\sqrt{1+K_{\mathrm{NN}}}} .
\end{aligned}
$$

After some simplification this gives

$$
\begin{aligned}
Q_{\mathrm{NN}} \Phi_{0}= & -\frac{1}{\sqrt{1+K_{\mathrm{NN}}}} c K_{\mathrm{NN}} c X_{1} \sigma X_{2} \frac{B}{1+K_{\mathrm{DD}}} \\
& \times X_{3} \bar{\sigma} X_{4}\left(1+K_{\mathrm{NN}}\right) c \frac{1}{\sqrt{1+K_{\mathrm{NN}}}} \\
& -\frac{1}{\sqrt{1+K_{\mathrm{NN}}}} c K_{\mathrm{NN}} c X_{1} \sigma X_{2} K_{\mathrm{DD}} \frac{B}{1+K_{\mathrm{DD}}} \\
& \times X_{3} \bar{\sigma} X_{4}\left(1+K_{\mathrm{NN}}\right) c \frac{1}{\sqrt{1+K_{\mathrm{NN}}}} \\
& +\frac{1}{\sqrt{1+K_{\mathrm{NN}}}} c\left(1+K_{\mathrm{NN}}\right) X_{1} \sigma X_{2} \frac{K_{\mathrm{DD}}}{1+K_{\mathrm{DD}}} \\
& \times X_{3} \bar{\sigma} X_{4}\left(1+K_{\mathrm{NN}}\right) c \frac{1}{\sqrt{1+K_{\mathrm{NN}}}} \\
& -\frac{1}{\sqrt{1+K_{\mathrm{NN}}}} c\left(1+K_{\mathrm{NN}}\right) X_{1} \sigma X_{2} \frac{B}{1+K_{\mathrm{DD}}} K_{\mathrm{DD}} \\
& \times X_{3} \bar{\sigma} X_{4} c K_{\mathrm{NN}} c \frac{1}{\sqrt{1+K_{\mathrm{NN}}}} \\
& -\frac{1}{\sqrt{1+K_{\mathrm{NN}}}} c\left(1+K_{\mathrm{NN}}\right) X_{1} \sigma X_{2} \frac{B}{1+K_{\mathrm{DD}}} \\
& \times X_{3} \bar{\sigma} X_{4} c K_{\mathrm{NN}} c \frac{1}{\sqrt{1+K_{\mathrm{NN}}}} .
\end{aligned}
$$

Similarly, using again the assumption that the $X$ are pure matter and also employing the OPE in (61) we can write

$$
\begin{aligned}
\left\{\Psi_{0}, \Phi_{0}\right\}= & \frac{1}{\sqrt{1+K_{\mathrm{NN}}}} c K_{\mathrm{NN}} c X_{1} \sigma X_{2} \frac{B}{1+K_{\mathrm{DD}}} \\
& \times X_{3} \bar{\sigma} X_{4}\left(1+K_{\mathrm{NN}}\right) c \frac{1}{\sqrt{1+K_{\mathrm{NN}}}} \\
& +\frac{1}{\sqrt{1+K_{\mathrm{NN}}}} c\left(1+K_{\mathrm{NN}}\right) X_{1} \sigma X_{2} \frac{B}{1+K_{\mathrm{DD}}} \\
& \times X_{3} \bar{\sigma} X_{4} c K_{\mathrm{NN}} c \frac{1}{\sqrt{1+K_{\mathrm{NN}}}}
\end{aligned}
$$




$$
\begin{aligned}
\Phi_{0}^{2}= & \frac{1}{\sqrt{1+K_{\mathrm{NN}}}} c\left(1+K_{\mathrm{NN}}\right) X_{1} \sigma X_{2} B c \frac{1}{1+K_{\mathrm{DD}}} \\
& \times X_{3} \bar{\sigma} X_{4}\left(1+K_{\mathrm{NN}}\right) c \frac{1}{\sqrt{1+K_{\mathrm{NN}}}} \\
& -\frac{1}{\sqrt{1+K_{\mathrm{NN}}}} c\left(1+K_{\mathrm{NN}}\right) X_{1} \sigma X_{2} \frac{1}{1+K_{\mathrm{DD}}} \\
& \times B c X_{3} \bar{\sigma} X_{4} c\left(1+K_{\mathrm{NN}}\right) \frac{1}{\sqrt{1+K_{\mathrm{NN}}}} .
\end{aligned}
$$

Substituting the results in Eqs. (103), (105), and (106), into the equation of motion of (30) and simplifying, we see that it is satisfied.

Since the equation of motion is satisfied, the energy is proportional to $\operatorname{Tr}\left[\Phi_{0}^{3}\right]$. Next, we calculate this trace. Let us replace $K_{\mathrm{NN}}$ by $K$ and $K_{\mathrm{DD}}$ by $\mathcal{K}$.

$$
\begin{aligned}
\operatorname{Tr}\left[\Phi^{3}\right]= & -\operatorname{Tr}\left[c(1+K) X_{1} \sigma X_{2} \frac{1}{1+\mathcal{K}}\right. \\
& \times X_{3} \bar{\sigma} X_{4}(1+K) B c \frac{1}{1+K} c(1+K) X_{1} \sigma X_{2} \frac{1}{1+\mathcal{K}} \\
& \times X_{3} \bar{\sigma} X_{4}(1+K) B c \frac{1}{1+K} c(1+K) X_{1} \sigma X_{2} \frac{1}{1+\mathcal{K}} \\
& \left.\times X_{3} \bar{\sigma} X_{4}(1+K) B c \frac{1}{1+K}\right] .
\end{aligned}
$$

If we replace $c(1+K)=(1+K) c-\partial c$ we obtain

$$
\begin{aligned}
\operatorname{Tr}\left[\Phi^{3}\right]= & \operatorname{Tr}\left[\partial c X_{1} \sigma X_{2} \frac{1}{1+\mathcal{K}}\right. \\
& \times X_{3} \bar{\sigma} X_{4}(1+K) B c \frac{1}{1+K} \partial c X_{1} \sigma X_{2} \frac{1}{1+\mathcal{K}} \\
& \times X_{3} \bar{\sigma} X_{4}(1+K) B c \frac{1}{1+K} \partial c \\
& \left.\times X_{1} \sigma X_{2} \frac{1}{1+\mathcal{K}} X_{3} \bar{\sigma} X_{4}(1+K) B c \frac{1}{1+K}\right]
\end{aligned}
$$

where we have used $c^{2}=0$. Using $B c+c B=1$, repeatedly, this can be further simplified as

$$
\begin{aligned}
\operatorname{Tr}\left[\Phi^{3}\right]= & \operatorname{Tr}\left[X_{1} \sigma X_{2} \partial c \frac{B}{1+\mathcal{K}} X_{3} \bar{\sigma} X_{4} X_{1} \sigma X_{2} \partial c \frac{1}{1+\mathcal{K}}\right. \\
& \times X_{3} \bar{\sigma} X_{4} X_{1} \sigma X_{2} \partial c \frac{1}{1+\mathcal{K}} \\
& \left.\times X_{3} \bar{\sigma} X_{4}(1+K) c \frac{1}{1+K}\right] .
\end{aligned}
$$

Recalling that $X_{3} \bar{\sigma} X_{4} X_{1} \sigma X_{2}=1$ we obtain

$$
\begin{aligned}
\operatorname{Tr}\left[\Phi^{3}\right]= & \operatorname{Tr}\left[\frac{B}{1+\mathcal{K}} \partial c \frac{1}{1+\mathcal{K}} \partial c \frac{1}{1+\mathcal{K}}\right. \\
& \left.\times X_{3} \bar{\sigma} X_{4}(1+K) c \frac{1}{1+K} X_{1} \sigma X_{2} \partial c\right],
\end{aligned}
$$

where we have also applied cyclic property of the trace. Replacing $(1+K) c=c(1+K)+\partial c$ we obtain

$$
\begin{aligned}
\operatorname{Tr}\left[\Phi^{3}\right]= & \operatorname{Tr}\left[\frac{B}{1+\mathcal{K}} \partial c \frac{1}{1+\mathcal{K}} \partial c \frac{1}{1+\mathcal{K}} c \partial c\right] \\
& +\operatorname{Tr}\left[\frac{B}{1+\mathcal{K}} \partial c \frac{1}{1+\mathcal{K}} \partial c \frac{1}{1+\mathcal{K}}\right. \\
& \left.\times X_{3} \bar{\sigma} X_{4} \partial c \frac{1}{1+K} X_{1} \sigma X_{2} \partial c\right] \\
= & \int_{0}^{\infty} \mathrm{d} t_{1} \mathrm{~d} t_{2} \mathrm{~d} t_{3} e^{-\left(t_{1}+t_{2}+t_{3}\right)} \\
& \times\left\langle\mathcal{B} \partial c(0) \partial c\left(t_{1}\right) c \partial c\left(t_{1}+t_{2}\right)\right\rangle_{t_{1}+t_{2}+t_{3}}\left(\langle 0 \mid 0\rangle_{\text {matter }}\right) \\
& +\int_{0}^{\infty} \mathrm{d} t_{1} \mathrm{~d} t_{2} \mathrm{~d} t_{3} \mathrm{~d} t_{4} e^{-\left(t_{1}+t_{2}+t_{3}+t_{4}\right)} \\
& \times\left\langle\mathcal{B} \partial c(0) \partial c\left(t_{1}\right) \partial c\left(t_{1}+t_{2}\right) \partial c\left(t_{1}+t_{2}+t_{3}\right)\right\rangle_{t_{1}+t_{2}+t_{3}+t_{4}} \\
& \times\left\langle X_{3} \bar{\sigma} X_{4}\left(t_{1}+t_{2}\right) X_{1} \sigma X_{2}\left(t_{1}+t_{2}+t_{3}\right)\right\rangle_{t_{1}+t_{2}+t_{3}+t_{4}},
\end{aligned}
$$

where we have assumed that the wedge states defined in terms of $\mathcal{K}$ are the same as those of $K$. The ghost part of the second integrand is zero, while the first gives

$$
\begin{aligned}
\operatorname{Tr}\left[\Phi_{0}^{3}\right]= & g_{*} \int_{0}^{\infty} \mathrm{d} x_{i} \frac{e^{-\left(t_{1}+t_{2}+t_{3}\right)}}{\pi}\left[\operatorname{Sin}\left(\frac{2 \pi\left(t_{1}+t_{2}\right)}{t_{1}+t_{2}+t_{3}}\right)\right. \\
& \left.-\operatorname{Sin}\left(\frac{2 \pi t_{1}}{t_{1}+t_{2}+t_{3}}\right)-\operatorname{Sin}\left(\frac{2 \pi t_{2}}{t_{1}+t_{2}+t_{3}}\right)\right] .
\end{aligned}
$$

Making the following usual change of variables:

$$
x=\frac{t_{1}}{t_{1}+t_{2}+t_{3}}, \quad y=\frac{t_{2}}{t_{1}+t_{2}+t_{3}}, \quad T=t_{1}+t_{2}+t_{3},
$$

we can write

$$
\begin{aligned}
& \operatorname{Tr}\left[\Phi_{0}^{3}\right]=-\frac{g_{*}}{\pi} \int_{0}^{\infty} \mathrm{d} T T^{2} e^{-T} \int_{0}^{1} \mathrm{~d} x \int_{0}^{1-x} \mathrm{~d} y \\
& \quad \times[\operatorname{Sin}(2 \pi x)+\operatorname{Sin}(2 \pi y)-\operatorname{Sin}(2 \pi(x+y))]=-\frac{3 g_{*}}{\pi^{2}} .
\end{aligned}
$$

Therefore,

$$
E=-\frac{1}{6} \operatorname{Tr}\left[\Phi_{0}^{3}\right]=\frac{g_{*}}{2 \pi^{2}} .
$$

\section{References}

1. M. Schnabl, Analytic solution for tachyon condensation in open string field theory. Adv. Theor. Math. Phys. 10, 433 (2006). arXiv:hep-th/0511286

2. I. Ellwood, M. Schanabl, Proof of vanishing cohomology at the tachyon vacuum. JHEP 0702, 096 (2007) 
3. E. Witten, Noncommutative geometry and string field theory. Nucl. Phys. B 268, 253 (1986)

4. Y. Okawa, Comments on Schnabl's analytic solution for tachyon condensation in Witten's open string field theory. JHEP 0604, 055 (2006). arXiv:hep-th/0603159

5. T. Erler, M. Schnabl, A simple analytic solution for tachyon condensation. JHEP 0910, 066 (2009). arXiv:0906.0979 [hep-th]

6. M. Kiermaier, Y. Okawa, L. Rastelli, B. Zwiebach, Analytic solutions for marginal deformations in open string field theory. arXiv:hep-th/0701249

7. Y. Okawa, Real analytic solutions for marginal deformations in open superstring field theory. arXiv:0704.3612 [hep-th]

8. M. Schnabl, Comments on marginal deformations in open string field theory. arXiv:hep-th/0701248

9. E. Fuchs, M. Kroyter, R. Potting, Marginal deformations in string field theory. arXiv:0704.2222 [hep-th]

10. B.H. Lee, C. Park, D.D. Tolla, Marginal deformations as lower dimensional D-brane solutions in open string field theory. arXiv:0710.1342 [hep-th]

11. M. Kiermaier, Y. Okawa, Exact marginality in open string field theory: a general framework. arXiv:0707.4472 [hep-th]

12. M. Kiermaier, Y. Okawa, P. Soler, Solutions from boundary condition changing operators in open string field theory. JHEP 1103, 122 (2011). arXiv:1009.6185 [hep-th]

13. C. Maccaferri, A simple solution for marginal deformations in open string field theory. JHEP 1405, 004 (2014). arXiv:1402.3546 [hepth]

14. A. Sen, Universality of the tachyon potential. JHEP 9912, 027 (1999). arXiv:hep-th/9911116

15. L. Bonora, C. Maccaferri, D.D. Tolla, Relevant deformations in open string field theory: a simple solution for lumps. JHEP 1111, 107 (2011). arXiv:1009.4158 [hep-th]

16. L. Bonora, S. Giaccari, D.D. Tolla, The energy of the analytic lump solution in SFT. JHEP 1108, 158 (2011). arXiv: 1105.5926 [hep-th]

17. L. Bonora, S. Giaccari, D.D. Tolla, Analytic solutions for Dp branes in SFT. JHEP 1112, 033 (2011). arXiv:1106.3914 [hep-th]

18. T. Erler, C. Maccaferri, String field theory solution for any open string background. JHEP 1410, 29 (2014). arXiv:1406.3021 [hepth]

19. E. Fuchs, M. Kroyter, Analytical solutions of open string field theory. Phys. Rep. 502, 89 (2011). arXiv:0807.4722 [hep-th]

20. M. Schnabl, Algebraic Solutions in Open String Field Theory-A Lightning Review. arXiv:1004.4858 [hep-th]

21. Y. Okawa, Analytic methods in open string field theory. Prog. Theor. Phys. 128, 1001-1060 (2012)

22. L. Bonora, String field theory: a short introduction. PoS ICMP 2013, 001 (2014)

23. T. Erler, C. Maccaferri, Maccaferri connecting solutions in open string field theory with singular gauge transformations. JHEP 1204, 107 (2012)

24. L. Cardy, Conformal invariance and surface critical behavior. Nucl. Phys. B 240, 514 (1984)

25. L. Cardy, Effect of boundary conditions on the operator content of two-dimensional conformally invariant theories. Nucl. Phys. B 275, 200 (1986)

26. L. Cardy, Boundary conditions, fusion rules and the Verlinde formula. Nucl. Phys. B 324, 581 (1989)

27. A.B. Zamolodchikov, Conformal scalar field on the hyperelliptic curve and critical Ashkin-Teller multipoint correlation functions. Nucl. Phys. B 285, 481 (1987)

28. A. Hashimoto, Dynamics of Dirichlet-Neumann open strings on D-branes. Nucl. Phys. B 496, 243 (1997). arXiv:hep-th/9608127

29. E. Gava, K.S. Narain, M.H. Sarmadi, On the bound states of p- and (p+2)-branes. Nucl. Phys. B 504, 214 (1997). arXiv:hep-th/9704006
30. J. Frohlich, O. Grandjean, A. Recknagel, V. Schomerus, Fundamental strings in Dp-Dq brane systems. Nucl. Phys. B 583, 381 (2000). arXiv:hep-th/9912079

31. L. Dixon, D. Friedan, E. Martinec, S. Shenker, The conformal field theory of orbifolds. Nucl. Phys. B 282, 13 (1987)

32. E. Corrigan, D.B. Fairlie, Off-shell staes in dual resonance theory. Nucl. Phys. B 91, 527 (1975)

33. D. Olive, J. Scherk, Towards satisfactory scattering amplitudes for dual fermions. Nucl. Phys. B 64, 334 (1973)

34. J.H. Schwarz, Off-mass-shell dual amplitudes without ghosts. Nucl. Phys. B 65, 131 (1973)

35. D. Olive, P. Goddard, R.A. Smith, D.J. Olive, Evaluation of the scattering amplitude for four dual fermions. Nucl. Phys. B 67, 477 (1973)

36. J.H. Schwarz, Dual quark-gluon theory with dynamical color. Nucl. Phys. B 68, 221 (1974)

37. E.F. Corrigan, The scattering amplitude for four dual fermions. Nucl. Phys. B 69, 325 (1974)

38. J.H. Schwarz, C.C. Wu, Off-shell dual amplitudes. II. Nucl. Phys. B 72, 397 (1974)

39. M.B. Green, Locality and currents for the dual string. Nucl. Phys. B 103, 333 (1976)

40. A. LeClair, M.E. Peskin, C.R. Preitschopf, String field theory on the conformal plane. 1. Kinematical principles. Nucl. Phys. B 317, 411 (1989)

41. A. LeClair, M.E. Peskin, C.R. Preitschopf, String field theory on the conformal plane. 2. Generalized gluing. Nucl. Phys. B 317, 464 (1989)

42. J.A. Harvey, P. Kraus, D-branes as unstable lumps in bosonic open string field theory. JHEP 0004, 012 (2000). arXiv:hep-th/0002117

43. R. de Mello Koch, A. Jevicki, M. Mihailescu, R. Tatar, Lumps and p-branes in open string field theory. Phys. Lett. B 482, 249 (2000). arXiv:hep-th/0003031

44. N. Moeller, A. Sen, B. Zwiebach, D-branes as tachyon lumps in string field theory. JHEP 0008, 039 (2000). arXiv:hep-th/0005036

45. R. de Mello Koch, J.P. Rodrigues, Lumps in level truncated open string field theory. Phys. Lett. B 495, 237 (2000). arXiv:hep-th/0008053

46. L. Rastelli, A. Sen, B. Zwiebach, Classical solutions in string field theory around the tachyon vacuum. Adv. Theor. Math. Phys. 5, 393 (2002). arXiv:hep-th/0102112

47. D. Kutasov, M. Marino, G.W. Moore, Some exact results on tachyon condensation in string field theory. JHEP 0010, 045 (2000)

48. E. Witten, Some computations in background independent off-shell string theory. Phys. Rev. D 47, 3405 (1993)

49. L. Bonora, S. Giaccari, D.D. Tolla, Lump solutions in SFT. Complements. arXiv: 1109.4336 [hep-th]

50. I. Kishimoto, T. Takahashi, Vacuum structure around identity based solutions. Prog. Theor. Phys. 122, 385 (2009). arXiv:0904.1095 [hep-th]

51. S. Zeze, Tachyon potential in KBc subalgebra. Prog. Theor. Phys. 124, 567 (2010). arXiv:1004.4351 [hep-th]

52. S. Zeze, Regularization of identity based solution in string field theory. arXiv:1008.1104 [hep-th]

53. E.A. Arroyo, Comments on regularization of identity based solutions in string field theory. arXiv:1009.0198 [hep-th]

54. L. Bonora, S. Giaccari, Generalized states in SFT. EPJC 73, 2644 (2013) 